\title{
The Energy-Momentum Method for the Stability of Nonholonomic Systems
}

\author{
Dmitry V. Zenkov* \\ Department of Mathematics \\ The Ohio State University \\ Columbus, $\mathrm{OH} 43210$ \\ zenkov@math.ohio-state.edu
}

\author{
Anthony M. Bloch ${ }^{\dagger}$ \\ Department of Mathematics \\ University of Michigan \\ Ann Arbor, MI 48109 \\ abloch@math.lsa.umich.edu
}

Jerrold E. Marsden $\ddagger$

Control and Dynamical Systems

California Institute of Technology 107-81

Pasadena, CA 91125

marsden@cds.caltech.edu

this version: June 19, 1998

AMS Subject Classification: 70H05, 53C22

Dynamics and Stability of Systems 13, 123-166.

\section{Contents}

1 Introduction $\quad \mathbf{2}$

1.1 The Lagrange-d'Alembert Principle . . . . . . . . . . . . . . . 4

1.2 Symmetries . . . . . . . . . . . . . . . . 6

1.3 The Rolling Disk . . . . . . . . . . . . . . . . . 6

1.4 A Mathematical Example . . . . . . . . . . . . 7

1.5 The Roller Racer . . . . . . . . . . . . . . . . . . . . . . . 9 9

1.6 The Rattleback . . . . . . . . . . . . . . . . . . . . . . . 10

* Research partially supported by National Science Foundation PYI grant DMS-9496221 and AFOSR grant F49620-96-1-0100

${ }^{\dagger}$ Research partially supported by National Science Foundation PYI grant DMS-9496221, AFOSR grant F49620-96-1-0100, a Guggenheim Fellowship and the Institute for Advanced Study

${ }^{\ddagger}$ Research partially supported by the National Science Foundation 
2 The Equations of Motion of Nonholonomic Systems with Symmetries $\quad 12$

2.1 The Geometry of Nonholonomic Systems with Symmetry . . 12

2.2 The Energy-Momentum Method for Holonomic Systems . . . 21

3 The Pure Transport Case 23

3.1 The Nonholonomic Energy-Momentum Method . . . . . . . 23

3.2 Examples ...................... 26

4 The Non-Pure Transport Case 28

4.1 Center Manifold Theory in Stability Analysis . . . . . . . . . 29

4.2 The Mathematical Example . . . . . . . . . . . . . . . . 32

4.3 The Nonholonomic Energy-Momentum Method . . . . . . . . 35

4.4 The Roller Racer . . . . . . . . . . . . . . . . . . . . . . . . . 42

4.5 Nonlinear Stability by the Lyapunov-Malkin Method . . . . . 43

4.6 The Lyapunov-Malkin and the Energy-Momentum Methods . 46

4.7 The Rattleback . . . . . . . . . . . . . . . . . . . . . . 48

4.8 Conclusions . . . . . . . . . . . . . . . . . . . . 51

$\begin{array}{ll}\text { References } & 51\end{array}$

\begin{abstract}
In this paper we analyze the stability of relative equilibria of nonholonomic systems (that is, mechanical systems with nonintegrable constraints such as rolling constraints). In the absence of external dissipation, such systems conserve energy, but nonetheless can exhibit both neutrally stable and asymptotically stable, as well as linearly unstable relative equilibria. To carry out the stability analysis, we use a generalization of the energy-momentum method combined with the Lyapunov-Malkin theorem and the center manifold theorem. While this approach is consistent with the energy-momentum method for holonomic systems, it extends it in substantial ways. The theory is illustrated with several examples, including the the rolling disk, the roller racer, and the rattleback top.
\end{abstract}

\title{
1 Introduction
}

The main goal of this paper is to analyze the stability of relative equilibria for nonholonomic mechanical systems with symmetry using an energymomentum analysis for nonholonomic systems that is analogous to that for holonomic systems given in Simo, Lewis, and Marsden [1991]. The theory of 
the motion of nonholonomic systems, which are mechanical systems subject to nonintegrable constraints, typified by rolling constraints, is remarkably rich. We will follow the spirit of the paper by Bloch, Krishnaprasad, Marsden and Murray [1996], hereafter referred to as [BKMM]. We will illustrate our energy-momentum stability analysis with a low-dimensional model example, and then with several mechanical examples of interest including the falling disk, the roller racer, and the rattleback top.

As discussed in [BKMM] (and elsewhere), symmetries do not always lead to conservation laws as in the classical Noether theorem, but rather to an interesting momentum equation. This is one of the manifestations of the difference between the Euler-Lagrange equations for holonomic mechanical systems and the Lagrange-d'Alembert equations for nonholonomic systems, which are not variational in nature. Another behavior which does not occur in unconstrained Hamiltonian and Lagrangian systems is that even in the absence of external forces and dissipation, nonholonomic systems may (but need not) possess asymptotically stable relative equilibria. This phenomenon was already known in the last century; cf. Walker [1896].

The momentum equation has the structure of a parallel transport equation for the momentum corrected by additional terms. This parallel transport occurs in a certain vector bundle over shape space. In some instances such as the Routh problem of a sphere rolling inside a surface of revolution (see Zenkov [1995]) this equation is pure transport, and in fact is integrable (the curvature of the transporting connection is zero). This leads to nonexplicit conservation laws.

In other important instances, the momentum equation is partially integrable in a sense that we shall make precise. Our goal is to make use of, as far as possible, the energy momentum approach to stability for Hamiltonian systems. This method goes back to fundamental work of Routh (and many others in this era), and in more modern works, that of Arnold [1966] and Smale [1970], and Simo, Lewis and Marsden [1991] (see for example, Marsden [1992] for an exposition and additional references). Because of the nature of the momentum equation, the analysis we present is rather different in several important respects. In particular, our energy-momentum analysis varies according to the structure of the momentum equation and, correspondingly, we divide our analysis into several parts.

There is a large literature on nonholonomic systems and here we shall cite only a small part of it. For a more comprehensive listing, see Neimark and Fufaev [1972] and [BKMM]. A key work on stability from our point of view may be found in Karapetyan [1980, 1983], which we shall specifically refer to in the course of this paper. Other work on symmetry and conservation laws 
my be found in Arnold [1988] and Bloch and Crouch [1992, 1995] for example. For the relationship between nonholonomic systems and Hamiltonian structures, see Bates and Sniatycki [1993] and Koon and Marsden [1997a, b, c] and references therein.

A brief of outline of this paper is as follows: In the remainder of this section we review the theory of nonholonomic systems and some key examples. In section 2 we discuss the role of symmetries in nonholonomic systems and the classical energy-momentum method for holonomic systems. In sections 3 and 4 we discuss the extension of the energy-momentum method to nonholonomic systems. As discussed above, we divide up the anaylsis into different parts according to the structure of the momentum equation. We list the underlying assumptions that we are making at the beginning of the relevant section. We note also that not all nonholonomic systems fall into the categories discussed here. We indicate in the main body of the paper precisely which systems are covered by our analysis.

\subsection{The Lagrange-d'Alembert Principle}

We now describe briefly the equations of motion for a nonholonomic system, following the notation of [BKMM].

We confine our attention to nonholonomic constraints that are homogeneous in the velocity. Accordingly, we consider a configuration space $Q$ and a distribution $\mathcal{D}$ that describes these constraints. Recall that a distribution $\mathcal{D}$ is a collection of linear subspaces of the tangent spaces of $Q$; we denote these spaces by $\mathcal{D}_{q} \subset T_{q} Q$, one for each $q \in Q$. A curve $q(t) \in Q$ will be said to satisfy the constraints if $\dot{q}(t) \in \mathcal{D}_{q(t)}$ for all $t$. This distribution will, in general, be nonintegrable; i.e., the constraints are, in general, nonholonomic.

Consider a Lagrangian $L: T Q \rightarrow \mathbb{R}$. In coordinates $q^{i}, i=1, \ldots, n$, on $Q$ with induced coordinates $\left(q^{i}, \dot{q}^{i}\right)$ for the tangent bundle, we write $L\left(q^{i}, \dot{q}^{i}\right)$. The equations of motion are given by the following Lagrange-d'Alembert principle.

Definition 1.1 The Lagrange-d'Alembert equations of motion for the system are those determined by

$$
\delta \int_{a}^{b} L\left(q^{i}, \dot{q}^{i}\right) d t=0
$$

where we choose variations $\delta q(t)$ of the curve $q(t)$ that satisfy $\delta q(a)=$ $\delta q(b)=0$ and $\delta q(t) \in \mathcal{D}_{q(t)}$ for each $t$ where $a \leq t \leq b$. 
This principle is supplemented by the condition that the curve itself satisfies the constraints. Note that we take the variation before imposing the constraints; that is, we do not impose the constraints on the family of curves defining the variation. This is well known to be important to obtain the correct mechanical equations (see [BKMM] for a discussion and references).

The usual arguments in the calculus of variations show that the Lagrange-d'Alembert principle is equivalent to the equations

$$
-\delta L=\left(\frac{d}{d t} \frac{\partial L}{\partial \dot{q}^{i}}-\frac{\partial L}{\partial q^{i}}\right) \delta q^{i}=0
$$

for all variations $\delta q$ such that $\delta q \in \mathcal{D}_{q}$ at each point of the underlying curve $q(t)$. One can of course equivalently write these equations in terms of Lagrange multipliers.

Let $\left\{\omega^{a}, a=1, \ldots, p\right\}$ be a set of $p$ independent one forms whose vanishing describes the constraints. Choose a local coordinate chart $q=(r, s) \in$ $\mathbb{R}^{n-p} \times \mathbb{R}^{p}$, which we write as $q^{i}=\left(r^{\alpha}, s^{a}\right)$, where $1 \leq \alpha \leq n-p$ and $1 \leq a \leq p$ such that

$$
\omega^{a}(q)=d s^{a}+A_{\alpha}^{a}(r, s) d r^{\alpha}
$$

for all $a=1, \ldots, p$. In these coordinates, the constraints are described by vectors $v^{i}=\left(v^{\alpha}, v^{a}\right)$ satisfying $v^{a}+A_{\alpha}^{a} v^{\alpha}=0$ (a sum on repeated indices over their range is understood).

The equations of motion for the system are given by (1.1) where we choose variations $\delta q(t)$ that satisfy the constraints, i.e., $\omega^{a}(q) \cdot \delta q=0$, or equivalently, $\delta s^{a}+A_{\alpha}^{a} \delta r^{\alpha}=0$, where $\delta q^{i}=\left(\delta r^{\alpha}, \delta s^{a}\right)$. Substituting variations of this type, with $\delta r^{\alpha}$ arbitrary, into (1.1) gives

$$
\left(\frac{d}{d t} \frac{\partial L}{\partial \dot{r}^{\alpha}}-\frac{\partial L}{\partial r^{\alpha}}\right)=A_{\alpha}^{a}\left(\frac{d}{d t} \frac{\partial L}{\partial \dot{s}^{a}}-\frac{\partial L}{\partial s^{a}}\right)
$$

for all $\alpha=1, \ldots, n-p$. Equation (1.2) combined with the constraint equations

$$
\dot{s}^{a}=-A_{\alpha}^{a} \dot{r}^{\alpha}
$$

for all $a=1, \ldots, p$ gives the complete equations of motion of the system.

A useful way of reformulating equations (1.2) is to define a "constrained" Lagrangian by substituting the constraints (1.3) into the Lagrangian:

$$
L_{c}\left(r^{\alpha}, s^{a}, \dot{r}^{\alpha}\right):=L\left(r^{\alpha}, s^{a}, \dot{r}^{\alpha},-A_{\alpha}^{a}(r, s) \dot{r}^{\alpha}\right) .
$$

The equations of motion can be written in terms of the constrained Lagrangian in the following way, as a direct coordinate calculation shows:

$$
\frac{d}{d t} \frac{\partial L_{c}}{\partial \dot{r}^{\alpha}}-\frac{\partial L_{c}}{\partial r^{\alpha}}+A_{\alpha}^{a} \frac{\partial L_{c}}{\partial s^{a}}=-\frac{\partial L}{\partial \dot{s}^{b}} B_{\alpha \beta}^{b} \dot{r}^{\beta},
$$


where $B_{\alpha \beta}^{b}$ is defined by

$$
B_{\alpha \beta}^{b}=\left(\frac{\partial A_{\alpha}^{b}}{\partial r^{\beta}}-\frac{\partial A_{\beta}^{b}}{\partial r^{\alpha}}+A_{\alpha}^{a} \frac{\partial A_{\beta}^{b}}{\partial s^{a}}-A_{\beta}^{a} \frac{\partial A_{\alpha}^{b}}{\partial s^{a}}\right) .
$$

Geometrically, the $A_{\alpha}^{a}$ are the coordinate expressions for the Ehresmann connection on the tangent bundle defined by the constraints, while the $B_{\alpha \beta}^{b}$ are the corresponding curvature terms (see $[\mathrm{BKMM}]){ }^{1}$

\section{$1.2 \quad$ Symmetries}

As we shall see shortly, symmetries play an important role in our analysis. We begin here with just a few preliminary notions. Suppose we are given a nonholonomic system with the Lagrangian $L: T Q \rightarrow \mathbb{R}$, and a (nonintegrable) constraint distribution $\mathcal{D}$. We can then look for a group $G$ that acts on the configuration space $Q$. It induces an action on the tangent space $T Q$ and so it makes sense to ask that the Lagrangian $L$ be invariant. Also, one can ask that the distribution be invariant in the sense that the action by a group element $g \in G$ maps the distribution $\mathcal{D}_{q}$ at the point $q \in Q$ to the distribution $\mathcal{D}_{g q}$ at the point $g q$. If these properties hold, we say that $G$ is a symmetry group.

In many examples, the symmetry group will be evident. For example, for systems rolling on the plane, the group of Euclidean motions of the plane, $S E(2)$ will be appropriate.

\subsection{The Rolling Disk}

A classical example of a nonholonomic system is a disk rolling without sliding on the $x y$-plane, as in Figure 1.1.

As the figure indicates, we denote the coordinates of contact of the disk in the $x y$-plane by $(x, y)$ and let $\theta, \phi$, and $\psi$ denote the angle between the plane of the disk and the vertical axis, the "heading angle" of the disk, and "self-rotation" angle of the disk respectively. In [BKMM], the vertical rolling disk was considered, but we consider a disk that can "fall".

A classical reference for the rolling disk is Vierkandt [1892] who showed that on the reduced space $\mathcal{D} / S E(2)$ - the constrained velocity phase space

\footnotetext{
${ }^{1}$ Strictly speaking, an Ehresmann connection requires one to have a bundle for which the distribution is regarded as the horizontal space but in fact, the bundle structure is not required if one regards the connection one form to be not vertical valued, but rather $T Q / \mathcal{D}$-valued.
} 


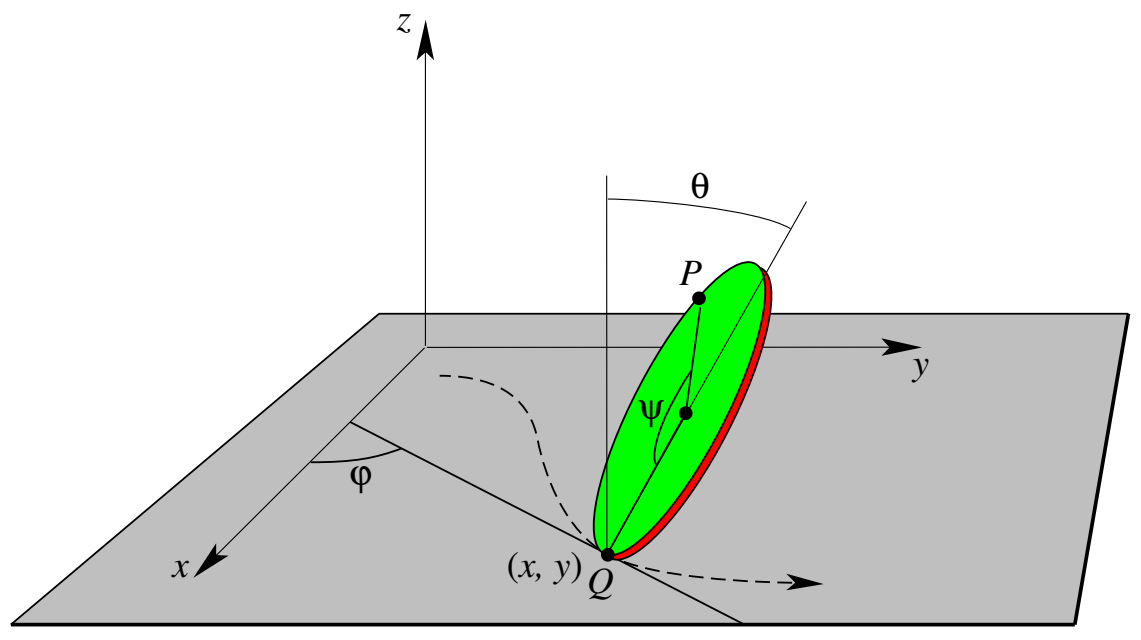

Figure 1.1: The geometry for the rolling disk.

modulo the action of the Euclidean group $S E(2)$ — most orbits of the system are periodic. Modern references that treat this example are Hermans [1995] and O'Reilly [1996].

For the moment, we just give the Lagrangian and constraints, and return to this example later on. As we will eventually show, this is a system which exhibits stability but not asymptotic stability. Denote the mass, the radius, and the moments of inertia of the disk by $m, R, A, B$ respectively. The Lagrangian is given by the kinetic minus potential energies:

$$
\begin{aligned}
L=\frac{m}{2} & {\left[(\xi-R(\dot{\phi} \sin \theta+\dot{\psi}))^{2}+\eta^{2} \sin ^{2} \theta+(\eta \cos \theta+R \dot{\theta})^{2}\right] } \\
& +\frac{1}{2}\left[A\left(\dot{\theta}^{2}+\dot{\phi}^{2} \cos ^{2} \theta\right)+B(\dot{\phi} \sin \theta+\dot{\psi})^{2}\right]-m g R \cos \theta,
\end{aligned}
$$

where $\xi=\dot{x} \cos \phi+\dot{y} \sin \phi+R \dot{\psi}$ and $\eta=-\dot{x} \sin \phi+\dot{y} \cos \phi$, while the constraints are given by

$$
\begin{aligned}
& \dot{x}=-\dot{\psi} R \cos \phi, \\
& \dot{y}=-\dot{\psi} R \sin \phi .
\end{aligned}
$$

Note that the constraints may also be written as $\xi=0, \eta=0$.

\subsection{A Mathematical Example}

We now consider an instructive, but (so far as we know) nonphysical example. Unlike the rolling disk, it has asymptotically stable relative equilibria, 
and is a simple example that exhibits the richness of stability in nonholonomic systems. Our general theorems presented later are well illustrated by this example and the reader may find it helpful to return to it again later.

Consider a Lagrangian on $T \mathbb{R}^{3}$ of the form

$$
\begin{aligned}
L\left(r^{1}, r^{2}, s, \dot{r}^{1}, \dot{r}^{2}, \dot{s}\right)=\frac{1}{2}\{(1- & {\left.\left[a\left(r^{1}\right)\right]^{2}\right)\left(\dot{r}^{1}\right)^{2}-2 a\left(r^{1}\right) b\left(r^{1}\right) \dot{r}^{1} \dot{r}^{2} } \\
& \left.+\left(1-\left[b\left(r^{1}\right)\right]^{2}\right)\left(\dot{r}^{2}\right)^{2}+\dot{s}^{2}\right\}-V\left(r^{1}\right),
\end{aligned}
$$

where $a, b$, and $V$ are given real valued functions of a single variable. We consider the nonholonomic constraint

$$
\dot{s}=a\left(r^{1}\right) \dot{r}^{1}+b\left(r^{1}\right) \dot{r}^{2} .
$$

Using the definitions, straightforward computations show that $B_{12}=$ $\partial_{r^{1}} b=-B_{21}$. The constrained Lagrangian is $\left.L_{c}=\frac{1}{2}\left\{\left(\dot{r}^{1}\right)^{2}+\left(\dot{r}^{2}\right)^{2}\right)\right\}-V\left(r^{1}\right)$ and the equations of motion, namely, $(d / d t)\left(\partial_{\dot{r}^{\alpha}} L_{c}\right)-\partial_{r^{\alpha}} L_{c}=-\dot{s} B_{\alpha \beta} \dot{r}^{\beta}$ become

$$
\frac{d}{d t} \frac{\partial L_{c}}{\partial \dot{r}^{1}}-\frac{\partial L_{c}}{\partial r^{1}}=-\dot{s} B_{12} \dot{r}^{2}, \quad \frac{d}{d t} \frac{\partial L_{c}}{\partial \dot{r}^{2}}=\dot{s} B_{12} \dot{r}^{1} .
$$

The Lagrangian is independent of $r^{2}$ and correspondingly, we introduce the nonholonomic momentum defined by

$$
p=\frac{\partial L_{c}}{\partial \dot{r}^{2}}
$$

We shall review the nonholonomic momentum later on in connection with general symmetries, but for now just regard this as a definition. Taking into account the constraint equation and the equations of motion above, we can rewrite the equations of motion in the form

$$
\begin{aligned}
\ddot{r}^{1} & =-\frac{\partial V}{\partial r^{1}}-\frac{\partial b}{\partial r^{1}}\left(a\left(r^{1}\right) \dot{r}^{1}+b\left(r^{1}\right) p\right) p, \\
\dot{p} & =\frac{\partial b}{\partial r^{1}}\left(a\left(r^{1}\right) \dot{r}^{1}+b\left(r^{1}\right) p\right) \dot{r}^{1} .
\end{aligned}
$$

Observe that the momentum equation does not, in any obvious way, imply a conservation law.

A relative equilibrium is a point $\left(r_{0}, p_{0}\right)$ that is an equilibrium modulo the variable $r^{2}$; thus, from the equations (1.6) and (1.7), we require $\dot{r}^{1}=0$ and

$$
\frac{\partial V}{\partial r^{1}}\left(r_{0}^{1}\right)+\frac{\partial b}{\partial r^{1}} b\left(r_{0}^{1}\right) p_{0}^{2}=0 .
$$

We shall see that relative equilibria are Lyapunov stable and in addition asymptotically stable in certain directions if the following two stability conditions are satisfied: 
(i) the energy function $E=\frac{1}{2}\left(\dot{r}^{1}\right)^{2}+\frac{1}{2} p^{2}+V$, which has a critical point at $\left(r_{0}, p_{0}\right)$, has a positive definite second derivative at this point.

(ii) the derivative of $E$ along the flow of the auxilliary system

$$
\ddot{r}^{1}=-\frac{\partial V}{\partial r^{1}}-\frac{\partial b}{\partial r^{1}}\left(a\left(r^{1}\right) \dot{r}^{1}+b\left(r^{1}\right) p\right) p, \quad \dot{p}=\frac{\partial b}{\partial r^{1}} b\left(r^{1}\right) p \dot{r}^{1}
$$

is strictly negative.

\subsection{The Roller Racer}

We now consider a tricycle-like mechanical system called the roller racer, or the Tennessee racer, that is capable of locomotion by oscillating the front handlebars. This toy was studied using the methods of [BKMM] in Tsakiris [1995]. The methods here may be useful for modeling and studying the stability of other systems, such as aircraft landing gears and train wheels.

The roller racer is modeled as a system of two planar coupled rigid bodies (the main body and the second body) with a pair of wheels attached on each of the bodies at their centers of mass. We assume that the mass and the linear momentum of the second body are negligible, but that the moment of inertia about the vertical axis is not. See Figure 1.2.

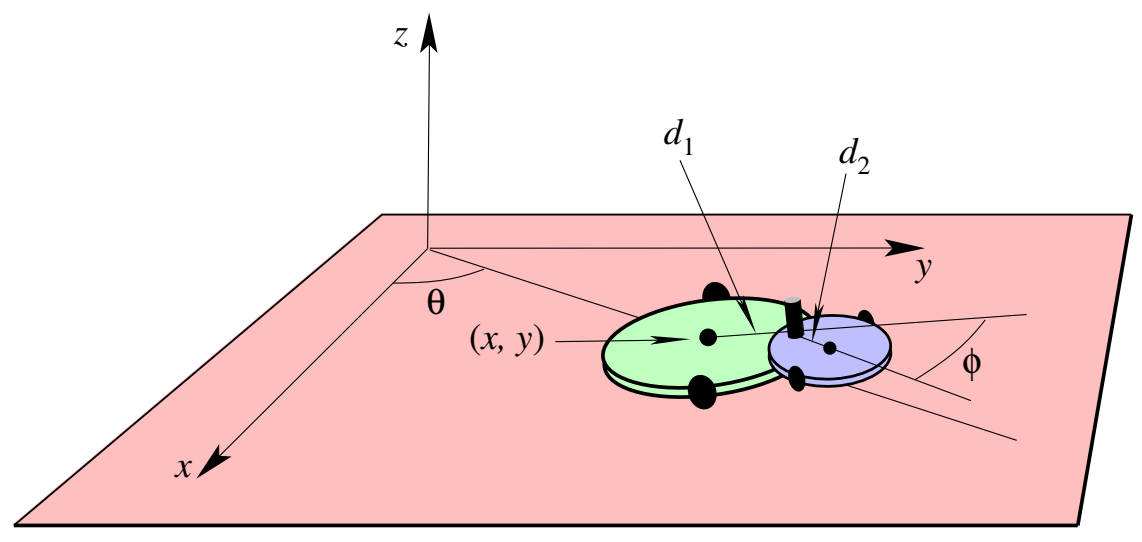

Figure 1.2: The geometry for the roller racer.

Let $(x, y)$ be the location of the center of mass of the first body and denote the angle between the $x$-axis of the inertial reference frame and the line passing through the center of mass of the first body by $\theta$, the angle between the bodies by $\phi$, and the distances from the centers of mass to the 
joint by $d_{1}$ and $d_{2}$. The mass of body 1 is denoted $m$ and the inertias of the two bodies are written as $I_{1}$ and $I_{2}$.

The Lagrangian and the constraints are

$$
L=\frac{1}{2} m\left(\dot{x}^{2}+\dot{y}^{2}\right)+\frac{1}{2} I_{1} \dot{\theta}^{2}+\frac{1}{2} I_{2}(\dot{\theta}+\dot{\phi})^{2}
$$

and

$$
\begin{aligned}
& \dot{x}=\cos \theta\left(\frac{d_{1} \cos \phi+d_{2}}{\sin \phi} \dot{\theta}+\frac{d_{2}}{\sin \phi} \dot{\phi}\right), \\
& \dot{y}=\sin \theta\left(\frac{d_{1} \cos \phi+d_{2}}{\sin \phi} \dot{\theta}+\frac{d_{2}}{\sin \phi} \dot{\phi}\right) .
\end{aligned}
$$

The configuration space is $S E(2) \times S O(2)$. The Lagrangian and the constraints are invariant under the left action of $S E(2)$ on the first factor of the configuration space.

We shall see later that the roller racer has a two-dimensional manifold of equilibria and that under a suitable stability condition some of these equilibria are stable modulo $S E(2)$ and in addition asymptotically stable with respect to $\dot{\phi}$.

\subsection{The Rattleback}

A rattleback is a convex nonsymmetric rigid body rolling without sliding on a horizontal plane. It is known for its ability to spin in one direction and to resist spinning in the opposite direction for some parameter values, and for other values, to exhibit multiple reversals. See Figure 1.3.

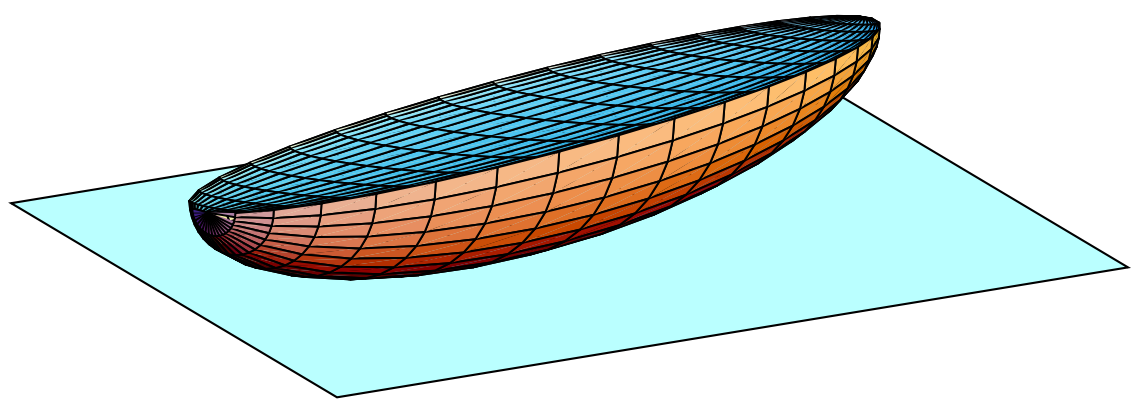

Figure 1.3: The rattleback.

Basic references on the rattleback are Walker [1896], Karapetyan [1980, 1981], Markeev [1983, 1992], Pascal [1983, 1986], and Bondi [1986]. We 
adopt the ideal model (with no energy dissipation and no sliding) of these references and within that context, no approximations are made. In particular, the shape need not be ellipsoidal. Walker did some initial stability and instability investigations by computing the spectrum while Bondi extended this analysis and also used what we now recognize as the momentum equation. (See Burdick, Goodwine and Ostrowski [1994]). Karapetyan carried out a stability analysis of the relative equilibria, while Markeev's and Pascal's main contributions were to the study of spin reversals using small parameter and averaging techniques.

Introduce the Euler angles $\theta, \phi, \psi$ using the principal axis body frame relative to an inertial reference frame. These angles together with two horizontal coordinates $(x, y)$ of the center of mass are coordinates in the configuration space $S O(3) \times \mathbb{R}^{2}$ of the rattleback.

The Lagrangian of the rattleback is computed to be

$$
\begin{aligned}
L= & \frac{1}{2}\left[A \cos ^{2} \psi+B \sin ^{2} \psi+m\left(\gamma_{1} \cos \theta-\zeta \sin \theta\right)^{2}\right] \dot{\theta}^{2} \\
& +\frac{1}{2}\left[\left(A \sin ^{2} \psi+B \cos ^{2} \psi\right) \sin ^{2} \theta+C \cos ^{2} \theta\right] \dot{\phi}^{2} \\
& +\frac{1}{2}\left(C+m \gamma_{2}^{2} \sin ^{2} \theta\right) \dot{\psi}^{2}+\frac{1}{2} m\left(\dot{x}^{2}+\dot{y}^{2}\right) \\
& +m\left(\gamma_{1} \cos \theta-\zeta \sin \theta\right) \gamma_{2} \sin \theta \dot{\theta} \dot{\psi}+(A-B) \sin \theta \sin \psi \cos \psi \dot{\theta} \dot{\phi} \\
& +C \cos \theta \dot{\phi} \dot{\psi}+m g\left(\gamma_{1} \sin \theta+\zeta \cos \theta\right),
\end{aligned}
$$

where

$A, B, C=$ the principal moments of inertia of the body,

$m=$ the total mass of the body,

$(\xi, \eta, \zeta)=$ coordinates of the point of contact relative to the body frame,

$\gamma_{1}=\xi \sin \psi+\eta \cos \psi$,

$\gamma_{2}=\xi \cos \psi-\eta \sin \psi$.

The shape of the body is encoded by the functions $\xi, \eta$ and $\zeta$. The constraints are

$$
\dot{x}=\alpha_{1} \dot{\theta}+\alpha_{2} \dot{\psi}+\alpha_{3} \dot{\phi}, \quad \dot{y}=\beta_{1} \dot{\theta}+\beta_{2} \dot{\psi}+\beta_{3} \dot{\phi},
$$


where

$$
\begin{aligned}
& \alpha_{1}=-\left(\gamma_{1} \sin \theta+\zeta \cos \theta\right) \sin \phi, \\
& \alpha_{2}=\gamma_{2} \cos \theta \sin \phi+\gamma_{1} \cos \phi, \\
& \alpha_{3}=\gamma_{2} \sin \phi+\left(\gamma_{1} \cos \theta-\zeta \sin \theta\right) \cos \phi, \\
& \beta_{k}=-\frac{\partial \alpha_{k}}{\partial \phi}, \quad k=1,2,3 .
\end{aligned}
$$

The Lagrangian and the constraints are $S E(2)$-invariant, where the action of an element $(a, b, \alpha) \in S E(2)$ is given by

$$
(x, y, \phi) \mapsto(x \cos \alpha-y \sin \alpha+a, x \sin \alpha+y \cos \alpha+b, \phi+\alpha) .
$$

Corresponding to this invariance, $\xi, \eta$, and $\zeta$ are functions of the variables $\theta$ and $\psi$ only.

\section{The Equations of Motion of Nonholonomic Sys- tems with Symmetries}

In this section we briefly discuss the mechanics of nonholonomic systems with symmetries - this of course is key to our energy momentum approach to stability. We make use of the approach introduced in [BKMM]. The key equations are (2.3) and (2.4) in a body frame, given below. It is the form of these equations that determines the stability and dynamics of the systems analyzed here. The knowledgable reader may skip directly to these equations at this point - the following text explains their derivation.

\subsection{The Geometry of Nonholonomic Systems with Symme- try}

Consider a nonholonomic system with the Lagrangian $L: T Q \rightarrow \mathbb{R}$, the (nonintegrable) constraint distribution $\mathcal{D}$, and the symmetry group $G$ in the sense explained previously.

Orbits and Shape Space. The group orbit through a point $q$, an (immersed) submanifold, is denoted

$$
\operatorname{Orb}(q):=\{g q \mid g \in G\} .
$$

Let $\mathfrak{g}$ denote the Lie algebra of the Lie group $G$. For an element $\xi \in \mathfrak{g}$, we write $\xi_{Q}$, a vector field on $Q$ for the corresponding infinitesimal generator. 
The tangent space to the group orbit through a point $q$ is given by the set of infinitesimal generators at that point:

$$
T_{q}(\operatorname{Orb}(q))=\left\{\xi_{Q}(q) \mid \xi \in \mathfrak{g}\right\} .
$$

Throughout this paper, we make the assumption that the action of $G$ on $Q$ is free and proper. The quotient space $M=Q / G$, whose points are the group orbits, is called shape space. Under these circumstances, shape space is a smooth manifold and the projection map $\pi: Q \rightarrow Q / G$ is a smooth surjective map with a surjective derivative $T_{q} \pi$ at each point. The kernel of the linear map $T_{q} \pi$ is the set of infinitesimal generators of the group action at the point $q$, i.e.,

$$
\operatorname{ker} T_{q} \pi=\left\{\xi_{Q}(q) \mid \xi \in \mathfrak{g}\right\},
$$

so these are also the tangent spaces to the group orbits.

Reduced Dynamics. Assuming that the Lagrangian and the constraint distribution are $G$-invariant, we can form the reduced velocity phase space $T Q / G$ and the reduced constraint space $\mathcal{D} / G$. The Lagrangian $L$ induces well defined functions, the reduced Lagrangian

$$
l: T Q / G \rightarrow \mathbb{R}
$$

satisfying $L=l \circ \pi_{T Q}$ where $\pi_{T Q}: T Q \rightarrow T Q / G$ is the projection, and the constrained reduced Lagrangian

$$
l_{c}: \mathcal{D} / G \rightarrow \mathbb{R},
$$

which satisfies $\left.L\right|_{\mathcal{D}}=l_{c} \circ \pi_{\mathcal{D}}$ where $\pi_{\mathcal{D}}: \mathcal{D} \rightarrow \mathcal{D} / G$ is the projection. By general considerations, the Lagrange-d'Alembert equations induce well defined reduced Lagrange-d'Alembert equations on $\mathcal{D} / G$. That is, the vector field on the manifold $\mathcal{D}$ determined by the Lagrange-d'Alembert equations (including the constraints) is $G$-invariant, and so defines a reduced vector field on the quotient manifold $\mathcal{D} / G$.

The nonholonomic momentum map. Consider the vector bundle $\mathcal{S}$ over $Q$ with fibers

$$
\mathcal{S}_{q}=\mathcal{D}_{q} \cap T_{q}(\operatorname{Orb}(q))
$$

Define, for each $q \in Q$, the vector subspace $\mathfrak{g}^{q}$ to be the set of Lie algebra elements in $\mathfrak{g}$ whose infinitesimal generators evaluated at $q$ lie in $\mathcal{S}_{q}$ :

$$
\mathfrak{g}^{q}=\left\{\xi \in \mathfrak{g} \mid \xi_{Q}(q) \in \mathcal{S}_{q}\right\} .
$$


The corresponding bundle over $Q$ whose fiber at the point $q$ is given by $\mathfrak{g}^{q}$, is denoted $\mathfrak{g}^{\mathcal{D}}$. Consider a section of the vector bundle $\mathcal{S}$ over $Q$; i.e., a mapping that takes $q$ to an element of $\mathcal{S}_{q}=\mathcal{D}_{q} \cap T_{q}(\operatorname{Orb}(q))$. Assuming that the action is free, a section of $\mathcal{S}$ can be uniquely represented as $\xi_{Q}^{q}$ and defines a section $\xi^{q}$ of the bundle $\mathfrak{g}^{\mathcal{D}}$.

Definition 2.1 The nonholonomic momentum map $J^{\text {nhc }}$ is the bundle map taking $T Q$ to the bundle $\left(\mathfrak{g}^{\mathcal{D}}\right)^{*}$ whose fiber over the point $q$ is the dual of the vector space $\mathfrak{g}^{q}$ that is defined by

$$
\left\langle J^{\mathrm{nhc}}\left(v_{q}\right), \xi\right\rangle=\frac{\partial L}{\partial \dot{q}^{i}}\left(\xi_{Q}\right)^{i},
$$

where $\xi \in \mathfrak{g}^{q}$. Intrinsically, this reads

$$
\left\langle J^{\mathrm{nhc}}\left(v_{q}\right), \xi\right\rangle=\left\langle\mathbb{F} L\left(v_{q}\right), \xi_{Q}\right\rangle,
$$

where $\mathbb{F} L$ is the fiber derivative of $L$ and where $\xi \in \mathfrak{g}^{q}$. For notational convenience, especially when the variable $v_{q}$ is suppressed, we will often write the left-hand side of this equation as $J^{\mathrm{nhc}}(\xi)$.

Notice that the nonholonomic momentum map may be viewed as giving just some of the components of the ordinary momentum map, namely along those symmetry directions that are consistent with the constraints.

For a nonholonomic system, the momentum map need not be a constant of motion. The following theorem states instead that the momentum map satisfies a certain equation.

Theorem 2.2 Assume that the Lagrangian and the constraint distribution are $G$-invariant, and that $\xi^{q}$ is a section of the bundle $\mathfrak{g}^{\mathcal{D}}$. Then any solution of the Lagrange-d'Alembert equations for a nonholonomic system must satisfy, in addition to the given kinematic constraints, the momentum equation:

$$
\frac{d}{d t}\left(J^{\mathrm{nhc}}\left(\xi^{q(t)}\right)\right)=\frac{\partial L}{\partial \dot{q}^{i}}\left[\frac{d}{d t}\left(\xi^{q(t)}\right)\right]_{Q}^{i} .
$$

When the momentum map is paired with a section in this way, we will just refer to it as the momentum.

The momentum in body representation. Let a local trivialization be chosen on the principle bundle $\pi: Q \rightarrow Q / G$, with a local representation having components denoted $(r, g)$. Let $r$, an element of shape space $Q / G$, 
have coordinates denoted $r^{\alpha}$, and let $g$ be group variables for the fiber, $G$. In such a representation, the action of $G$ is the left action of $G$ on the second factor.

Put

$$
l(r, \dot{r}, \xi)=L(r, g, \dot{r}, \dot{g}) .
$$

Choose a $q$-dependent basis $e_{a}(q)$ for the Lie algebra such that the first $m$ elements span the subspace $\mathfrak{g}^{q}$ in the following way. First, one chooses, for each $r$, such a basis at the identity element $g=\mathrm{Id}$, say

$$
e_{1}(r), e_{2}(r), \ldots, e_{m}(r), e_{m+1}(r), \ldots, e_{k}(r) .
$$

For example, this could be a basis whose generators are orthonormal in the kinetic energy metric. Now define the body fixed basis by

$$
e_{a}(r, g)=\operatorname{Ad}_{g} e_{a}(r)
$$

then the first $m$ elements will indeed span the subspace $\mathfrak{g}^{q}$ since the distribution is invariant. Define the components of the momentum in body representation to be

$$
p_{b}:=\left\langle\frac{\partial l}{\partial \xi}, e_{b}(r)\right\rangle
$$

Thus, we have

$$
J^{\mathrm{nhc}}(r, g, \dot{r}, \dot{g})=\operatorname{Ad}_{g^{-1}}^{*} p(r, \dot{r}, \xi) .
$$

This formula explains why $p$ and $J^{\text {nhc }}$ are the body momentum and the spatial momentum respectively.

The Nonholonomic Connection. Assume that the Lagrangian has the form kinetic energy minus potential energy, and that the constraints and the orbit directions span the entire tangent space to the configuration space ([BKMM] call this the "dimension assumption"):

$$
\mathcal{D}_{q}+T_{q}(\operatorname{Orb}(q))=T_{q} Q
$$

In this case, the momentum equation can be used to augment the constraints and provide a connection on $Q \rightarrow Q / G$.

Definition 2.3 Under the dimension assumption in equation (2.1), and the assumption that the Lagrangian is of the form kinetic minus potential energies, the nonholonomic connection $\mathcal{A}$ is the connection on the principal bundle $Q \rightarrow Q / G$ whose horizontal space at the point $q \in Q$ is given by the orthogonal complement to the space $\mathcal{S}_{q}$ within the space $\mathcal{D}_{q}$. 

by

Let $\mathbb{I}(q): \mathfrak{g}^{\mathcal{D}} \rightarrow\left(\mathfrak{g}^{\mathcal{D}}\right)^{*}$ be the locked inertia tensor relative to $\mathfrak{g}^{\mathcal{D}}$, defined

$$
\langle\mathbb{I}(q) \xi, \eta\rangle=\left\langle\left\langle\xi_{Q}, \eta_{Q}\right\rangle\right\rangle, \quad \xi, \eta \in \mathfrak{g}^{q},
$$

where $\langle\langle\cdot, \cdot\rangle\rangle$ is the kinetic energy metric. Define a map $A_{q}^{\text {sym }}: T_{q} Q \rightarrow \mathcal{S}_{q}=$ $\mathcal{D}_{q} \cap T_{q}(\operatorname{Orb}(q))$ given by

$$
A_{q}^{\mathrm{sym}}\left(v_{q}\right)=\left(\mathbb{I}^{-1} J^{\mathrm{nhc}}\left(v_{q}\right)\right)_{Q} .
$$

This map is equivariant and is a projection onto $\mathcal{S}_{q}$. Choose $\mathcal{U}_{q} \subset T_{q}(\mathrm{Orb}(q))$ such that $T_{q}(\operatorname{Orb}(q))=\mathcal{S}_{q} \oplus \mathcal{U}_{q}$. Let $A_{q}^{\text {kin }}: T_{q} Q \rightarrow \mathcal{U}_{q}$ be a $\mathcal{U}_{q}$ valued form that projects $\mathcal{U}_{q}$ onto itself and maps $\mathcal{D}_{q}$ to zero; for example, it can be given by orthogonal projection relative to the kinetic energy metric (this will be our default choice).

Proposition 2.4 The nonholonomic connection regarded as an Ehresmann connection is given by

$$
A=A^{\mathrm{kin}}+A^{\mathrm{sym}} .
$$

When the connection is regarded as a principal connection (i.e., takes values in the Lie algebra rather than the vertical space) we will use the symbol $\mathcal{A}$.

Given a velocity vector $\dot{q}$ that satisfies the constraints, we orthogonally decompose it into a piece in $\mathcal{S}_{q}$ and an orthogonal piece denoted $\dot{r}^{h}$. We regard $\dot{r}^{h}$ as the horizontal lift of a velocity vector $\dot{r}$ on the shape space; recall that in a local trivialization, the horizontal lift to the point $(r, g)$ is given by

$$
\dot{r}^{h}=\left(\dot{r},-\mathcal{A}_{\mathrm{loc}} \dot{r}\right)=\left(\dot{r}^{\alpha},-\mathcal{A}_{\alpha}^{a} \dot{r}^{\alpha}\right),
$$

where $\mathcal{A}_{\alpha}^{a}$ are the components of the nonholonomic connection which is a principal connection in a local trivialization.

We will denote the decomposition of $\dot{q}$ by

$$
\dot{q}=\Omega_{Q}(q)+\dot{r}^{h},
$$

so that for each point $q, \Omega$ is an element of the Lie algebra and represents the spatial angular velocity of the locked system. In a local trivialization, we can write, at a point $(r, g)$,

$$
\Omega=\operatorname{Ad}_{g}\left(\Omega_{\mathrm{loc}}\right),
$$

so that $\Omega_{\text {loc }}$ represents the body angular velocity. Thus,

$$
\Omega_{\mathrm{loc}}=\mathcal{A}_{\mathrm{loc}} \dot{r}+\xi
$$


and, at each point $q$, the constraints are that $\Omega$ belongs to $\mathfrak{g}^{q}$, i.e.,

$$
\Omega_{\mathrm{loc}} \in \operatorname{span}\left\{e_{1}(r), e_{2}(r), \ldots, e_{m}(r)\right\} .
$$

The vector $\dot{r}^{h}$ need not be orthogonal to the whole orbit, just to the piece $\mathcal{S}_{q}$. Even if $\dot{q}$ does not satisfy the constraints, we can decompose it into three parts and write

$$
\dot{q}=\Omega_{Q}(q)+\dot{r}^{h}=\Omega_{Q}^{\mathrm{nh}}(q)+\Omega_{Q}^{\perp}(q)+\dot{r}^{h},
$$

where $\Omega_{Q}^{\text {nh }}$ and $\Omega_{Q}^{\perp}$ are orthogonal and where $\Omega_{Q}^{\mathrm{nh}}(q) \in \mathcal{S}_{q}$. The relation $\Omega_{\text {loc }}=\mathcal{A}_{\text {loc }} \dot{r}+\xi$ is valid even if the constraints do not hold; also note that this decomposition of $\Omega$ corresponds to the decomposition of the nonholonomic connection given by $A=A^{\mathrm{kin}}+A^{\mathrm{sym}}$ that was given in equation (2.2).

To avoid confusion, we will make the following index and summation conventions

1. The first batch of indices range from 1 to $m$ corresponding to the symmetry directions along constraint space. These indices will be denoted $a, b, c, d, \ldots$ and a summation from 1 to $m$ will be understood.

2. The second batch of indices range from $m+1$ to $k$ corresponding to the symmetry directions not aligned with the constraints. Indices for this range or for the whole range 1 to $k$ will be denoted by $a^{\prime}, b^{\prime}, c^{\prime}, \ldots$ and the summations will be given explicitly.

3. The indices $\alpha, \beta, \ldots$ on the shape variables $r$ range from 1 to $\sigma$. Thus, $\sigma$ is the dimension of the shape space $Q / G$ and so $\sigma=n-k$. The summation convention for these indices will be understood.

According to $[\mathrm{BKMM}]$, the equations of motion are given by the next theorem.

Theorem 2.5 The following reduced nonholonomic Lagrange-d'Alembert equations hold for each $1 \leq \alpha \leq \sigma$ and $1 \leq b \leq m$ :

$$
\begin{aligned}
\frac{d}{d t} \frac{\partial l_{c}}{\partial \dot{r}^{\alpha}}-\frac{\partial l_{c}}{\partial r^{\alpha}}= & -\frac{\partial I^{c d}}{\partial r^{\alpha}} p_{c} p_{d}-\mathcal{D}_{b \alpha}^{c} I^{b d} p_{c} p_{d}-\mathcal{B}_{\alpha \beta}^{c} p_{c} \dot{r}^{\beta} \\
& -\mathcal{D}_{\beta \alpha b} I^{b c} p_{c} \dot{r}^{\beta}-\mathcal{K}_{\alpha \beta \gamma} \dot{r}^{\beta} \dot{r}^{\gamma} \\
\frac{d}{d t} p_{b}= & C_{a b}^{c} I^{a d} p_{c} p_{d}+\mathcal{D}_{b \alpha}^{c} p_{c} \dot{r}^{\alpha}+\mathcal{D}_{\alpha \beta b} \dot{r}^{\alpha} \dot{r}^{\beta}
\end{aligned}
$$

Here $l_{c}\left(r^{\alpha}, \dot{r}^{\alpha}, p_{a}\right)$ is the constrained Lagrangian; $r^{\alpha}, 1 \leq \alpha \leq \sigma$, are coordinates in the shape space; $p_{a}, 1 \leq a \leq m$, are components of the momentum 
map in the body representation, $p_{a}=\left\langle\partial l_{c} / \partial \Omega_{\mathrm{loc}}, e_{a}(r)\right\rangle ; I^{\text {ad }}$ are the components of the inverse locked inertia tensor; $\mathcal{B}_{\alpha \beta}^{a}$ are the local coordinates of the curvature $\mathcal{B}$ of the nonholonomic connection $\mathcal{A}$; and the coefficients $\mathcal{D}_{b \alpha}^{c}$, $\mathcal{D}_{\alpha \beta b}, \mathcal{K}_{\alpha \beta \gamma}$ are given by the formulae

$$
\begin{aligned}
\mathcal{D}_{b \alpha}^{c} & =\sum_{a^{\prime}=1}^{k}-C_{a^{\prime} b}^{c} \mathcal{A}_{\alpha}^{a^{\prime}}+\gamma_{b \alpha}^{c}+\sum_{a^{\prime}=m+1}^{k} \lambda_{a^{\prime} \alpha} C_{a b}^{a^{\prime}} I^{a c}, \\
\mathcal{D}_{\alpha \beta b} & =\sum_{a^{\prime}=m+1}^{k} \lambda_{a^{\prime} \alpha}\left(\gamma_{b \beta}^{a^{\prime}}-\sum_{b^{\prime}=1}^{k} C_{b^{\prime} b}^{a^{\prime}} \mathcal{A}_{\beta}^{b^{\prime}}\right) \\
\mathcal{K}_{\alpha \beta \gamma} & =\sum_{a^{\prime}=1}^{k} \lambda_{a^{\prime} \gamma} \mathcal{B}_{\alpha \beta}^{a^{\prime}},
\end{aligned}
$$

where

$$
\lambda_{a^{\prime} \alpha}=l_{a^{\prime} \alpha}-\sum_{b^{\prime}=1}^{k} l_{a^{\prime} b^{\prime}} \mathcal{A}_{\alpha}^{b^{\prime}}:=\frac{\partial l}{\partial \xi^{a^{\prime}} \partial \dot{r}^{\alpha}}-\sum_{b^{\prime}=1}^{k} \frac{\partial l}{\partial \xi^{a^{\prime}} \partial \xi^{b^{\prime}}} \mathcal{A}_{\alpha}^{b^{\prime}}
$$

for $a^{\prime}=m+1, \ldots, k$. Here $C_{a^{\prime} c^{\prime}}^{b^{\prime}}$ are the structure constants of the Lie algebra defined by $\left[e_{a^{\prime}}, e_{c^{\prime}}\right]=C_{a^{\prime} c^{\prime}}^{b^{\prime}} e_{b^{\prime}}, a^{\prime}, b^{\prime}, c^{\prime}=1, \ldots, k$; and the coefficients $\gamma_{b \alpha}^{c^{\prime}}$ are defined by

$$
\frac{\partial e_{b}}{\partial r^{\alpha}}=\sum_{c^{\prime}=1}^{k} \gamma_{b \alpha}^{c^{\prime}} e_{c^{\prime}}
$$

A relative equilibrium is an equilibrium of the reduced equations; that is, it is a solution that is given by a one parameter group orbit, just as in the holonomic case (see, e.g., Marsden [1992] for a discussion).

The Constrained Routhian. This function is defined by analogy with the usual Routhian by

$$
R\left(r^{\alpha}, \dot{r}^{\alpha}, p_{a}\right)=l_{c}\left(r^{\alpha}, \dot{r}^{\alpha}, I^{a b} p_{b}\right)-I^{a b} p_{a} p_{b},
$$

and in terms of it, the reduced equations of motion become

$$
\begin{aligned}
\frac{d}{d t} \frac{\partial R}{\partial \dot{r}^{\alpha}}-\frac{\partial R}{\partial r^{\alpha}}= & -\mathcal{D}_{b \alpha}^{c} I^{b d} p_{c} p_{d}-\mathcal{B}_{\alpha \beta}^{c} p_{c} \dot{r}^{\beta} \\
& -\mathcal{D}_{\beta \alpha b} I^{b c} p_{c} \dot{r}^{\beta}-\mathcal{K}_{\alpha \beta \gamma} \dot{r}^{\beta} \dot{r}^{\gamma} \\
\frac{d}{d t} p_{b}= & C_{a b}^{c} I^{a d} p_{c} p_{d}+\mathcal{D}_{b \alpha}^{c} p_{c} \dot{r}^{\alpha}+\mathcal{D}_{\alpha \beta b} \dot{r}^{\alpha} \dot{r}^{\beta}
\end{aligned}
$$


The Reduced Constrained Energy. As in [BKMM], the kinetic energy in the variables $\left(r^{\alpha}, \dot{r}^{\alpha}, \Omega^{a}, \Omega^{a^{\prime}}\right)$ equals

$$
\begin{aligned}
\frac{1}{2} g_{\alpha \beta} \dot{r}^{\alpha} \dot{r}^{\beta} & +\frac{1}{2} I_{a c} \Omega^{a} \Omega^{c} \\
& +\sum_{a^{\prime}=m+1}^{k}\left(l_{a^{\prime} \alpha}-l_{a^{\prime} c^{\prime}} \mathcal{A}_{\alpha}^{c^{\prime}}\right) \Omega^{a^{\prime}} \dot{r}^{\alpha}+\frac{1}{2} \sum_{a^{\prime}, c^{\prime}=m+1}^{k} l_{a^{\prime} c^{\prime}} \Omega^{a^{\prime}} \Omega^{c^{\prime}},
\end{aligned}
$$

where $g_{\alpha \beta}$ are coefficients of the kinetic energy metric induced on the manifold $Q / G$. Substituting the relations $\Omega^{a}=I^{a b} p_{b}$ and the constraint equations $\Omega^{a^{\prime}}=0$ in (2.5) and adding the potential energy, we define the function $E$ by

$$
E=\frac{1}{2} g_{\alpha \beta} \dot{r}^{\alpha} \dot{r}^{\beta}+U\left(r^{\alpha}, p_{a}\right),
$$

which represents the reduced constrained energy in the coordinates $(r, \dot{r}, p)$, where $U\left(r^{\alpha}, p_{a}\right)$ is the amended potential defined by

$$
U\left(r^{\alpha}, p_{a}\right)=\frac{1}{2} I^{a b} p_{a} p_{b}+V\left(r^{\alpha}\right)
$$

and $V\left(r^{\alpha}\right)$ is the potential energy of the system.

Now, we show that the reduced constrained energy is conserved along the solutions of $(2.3),(2.4)$.

Theorem 2.6 The reduced constrained energy is a constant of motion.

Proof One way to prove this is to note that the reduced energy is a constant of motion, because it equals the energy represented in coordinates $(r, \dot{r}, p, g)$ and because the energy is conserved, since the Lagrangian and the constraints are time-invariant. Along the trajectories, the constrained energy and the energy are the same. Therefore, the reduced constrained energy is a constant of motion.

One may also prove this fact by a direct computation of the time derivative of the reduced constrained energy (2.6) along the vector field defined by the equations of motion.

Skew Symmetry Assumption. We assume that the tensor $C_{a b}^{c} I^{a d}$ is skew-symmetric in $c, d$.

We remark firstly that this assumption implies that the dimension of the family of the relative equilibria equals the number of components of the (nonholonomic) momentum map. This is important for our energetic approach to the analysis of stability. 
We note also that this assumption holds for most physical examples and certainly the systems discussed in this paper. (Exceptions include systems with no shape space such as the homogeneous sphere on the plane and certain cases "of the "Suslov" problem of a nonhomogeneous rigid body subject to a linear constraint in the angular velocities.) It is an intrinsic (coordinate independent) condition, since $C_{a b}^{c} I^{a d}$ represents an intrinsic bilinear map of $\left(\mathfrak{g}^{\mathcal{D}}\right)^{*} \times\left(\mathfrak{g}^{\mathcal{D}}\right)^{*}$ to $\left(\mathfrak{g}^{\mathcal{D}}\right)^{*}$.

Under this assumption, the terms quadratic in $p$ in the momentum equation vanish, and the equations of motion become

$$
\begin{aligned}
\frac{d}{d t} \frac{\partial R}{\partial \dot{r}^{\alpha}}-\frac{\partial R}{\partial r^{\alpha}}= & -\mathcal{D}_{b \alpha}^{c} I^{b d} p_{c} p_{d}-\mathcal{B}_{\alpha \beta}^{c} p_{c} \dot{r}^{\beta} \\
& -\mathcal{D}_{\beta \alpha b} I^{b c} p_{c} \dot{r}^{\beta}-\mathcal{K}_{\alpha \beta \gamma} \dot{r}^{\beta} \dot{r}^{\gamma}, \\
\frac{d}{d t} p_{b}= & \mathcal{D}_{b \alpha}^{c} p_{c} \dot{r}^{\alpha}+\mathcal{D}_{\alpha \beta b} \dot{r}^{\alpha} \dot{r}^{\beta} .
\end{aligned}
$$

In the case when $C_{a b}^{c}=0$ the matrix $C_{a b}^{c} I^{a d}$ vanishes, and the preceding equations of motion are the same as those obtained by Karapetyan [1983]. Note that $C_{a b}^{c}=0$ when the indices $a, b, c$ range over an abelian part of the symmetry group. This occurs for all examples discussed here.

Below, three principal cases will be considered:

1. Pure Transport Case In this case, terms quadratic in $\dot{r}$ are not present in the momentum equation, so it is in the form of a transport equation - i.e. the momentum equation is an equation of parallel transport and the equation itself defines the relevant connection.

Under certain integrability conditions (see below) the transport equation defines invariant surfaces, which allow us to use a type of energymomentum method for stability analysis in a similar fashion to the manner in which the holonomic case uses the level surfaces defined by the momentum map. The key difference is that in our case, the additional invariant surfaces do not arise from conservation of momentum. In this case, one gets stable, but not asymptotically stable, relative equilibria as we shall see in $\S 3.1$. Examples include the rolling disk, a body of revolution rolling on a horizontal plane, and the Routh problem.

2. Integrable Transport Case In this case, terms quadratic in $\dot{r}$ are present in the momentum equation and thus it is not a pure transport equation. However, in this case, we assume that the transport 
part is integrable. As we shall also see, in this case relative equilibria may be asymptotically stable. We are able to find a generalization of the energy-momentum method which gives conditions for asymptotic stability (see §4.3). An example is the roller racer.

3. Nonintegrable Transport Case Again, the terms quadratic in $\dot{r}$ are present in the momentum equation and thus it is not a pure transport equation. However, the transport part is not integrable. Again, we are able to demonstrate asymptotic stability using Lyapunov-Malkin theorem and to relate it to an energy-momentum type analysis under certain eigenvalue hypotheses, as we will see in $\S 4.5$. An example is the rattleback top, which we discuss in $\S 4.7$. Another example is a nonhomogeneous sphere with a center of mass lying off the planes spanned by the principal axis body frame. See Markeev [1992].

In some examples, such as the nonhomogeneous (unbalanced) Kovalevskaya sphere rolling on the plane, these eigenvalue hypotheses do not hold. We intend to investigate this case in a future publication.

As indicated above, the key difference between cases 1 and 2 is the existence of the nontranport terms in the momentum equation. These terms cause the momentum to drift between the invariant manifolds that arise from

the integrable pure transport term. As a result, in case 2 relative equilibria may be asymptotically stable. Similar qualitative behavior occurs in case 3 where even the transport part of the momentum equation does not define invariant manifolds.

In the sections below where these different cases are discussed we will make clear at the beginning of each section what the underlying hypotheses on the systems are by listing the key hypotheses and labeling them by H1, $\mathrm{H} 2$, and $\mathrm{H} 3$.

\subsection{The Energy-Momentum Method for Holonomic Systems}

As mentioned above, we use here an approach to stability which generalizes the energy-momentum method for Hamiltonian systems. Of course the energy-momentum method has a long and distinguished history going back to Routh, Riemann, Poincaré, Lyapunov, Arnold, Smale and many others. The main new feature provided in the more recent work of Simo, Lewis and Marsden [1991] (see Marsden [1992] for an exposition) is to obtain the powerful block diagonalization structure of the second variation of the augmented Hamiltonian as well as the normal form for the symplectic structure. This 
formulation also allowed for the proof of a converse of the energy-momentum method in the context of dissipation induced instabilities due to Bloch, Krishnaprasad, Marsden and Ratiu [1994, 1996].

Recall that the key idea for analyzing the stability of relative equilibria in the holonomic setting is to use the energy plus a function of other conserved quantities such as the momentum as a Lyapunov function. In effect, one is analyzing stability subject to the systems lying on a level surface of the momentum.

In a body frame and in the special case of Lie-Poisson systems, the momentum often can be written in terms of a Casimir, a function that commutes with every function under the Poisson bracket, and the method is sometimes called the energy-Casimir method.

While the energy is conserved, it does not provide sufficient information on stability since its second variation will be only semidefinite at a stable equilibrium in general. The algorithm for analyzing stability is thus as follows:

1. write the equations of motion in Hamiltonian form and identify the critical point of interest,

2. identify other conserved quantities such as momentum,

3. choose a function $H_{C}$ such that the energy plus the function of other conserved quantities has a critical point at the chosen equilibrium and

4. show that $H_{C}$ is definite at the given equilibrium. This proves nonlinear stability in the sense of Lyapunov.

Of course in special circumstances one has to interpret stability modulo the symmetry or a similar space in order to obtain stability. A good example is the study of two-dimensional ABC flows, as in Chern and Marsden [1990].

In the nonholonomic case, while energy is conserved, momentum generally is not. As indicated in the discussion of the three principle cases above, in some cases however the momentum equation is integrable, leading to invariant surfaces which make possible an energy-momentum analysis similar to that of the Hamiltonian case. When the momentum equation is not integrable, one can get asymptotic stability in certain directions and the stability analysis is rather different from the Hamiltonian case. Nonetheless, to show stability we will make use of the conserved energy and the dynamic momentum equation. 


\section{The Pure Transport Case}

In this section we assume that

$\mathrm{H} 1 \mathcal{D}_{\alpha \beta b}$ are skew-symmetric in $\alpha, \beta$. Under this assumption, the momentum equation can be written as the vanishing of the connection one form defined by $d p_{b}-\mathcal{D}_{b \alpha}^{c} p_{c} d r^{\alpha}$.

$\mathrm{H} 2$ The curvature of the preceding connection form is zero.

A nontrivial example of this case is that of Routh's problem of a sphere rolling in a surface of revolution. See Zenkov [1995].

Under the above two assumptions, the distribution defined by the momentum equation is integrable, and so we get invariant surfaces, which makes further reduction possible. This enables us to use the energy-momentum method in a way that is similar to the holonomic case, as we explained above.

Note that if the number of shape variables is one, the above connection is integrable, because it may be treated as a system of linear ordinary differential equations with coefficients depending on the shape variable $r$ :

$$
\frac{d p_{b}}{d r}=\mathcal{D}_{b}^{c} p_{c}
$$

As a result, we obtain an integrable nonholonomic system, because after solving the momentum equation for $p_{b}$ and substituting the result in the equation for the shape variable, the latter equation may be viewed as a Lagrangian system with one degree of freedom, which is integrable.

\subsection{The Nonholonomic Energy-Momentum Method}

We now develop the energy-momentum method for the case in which the momentum equation is pure transport. Under the assumptions H1 and H2 made so far, the equations of motion become

$$
\begin{aligned}
\frac{d}{d t} \frac{\partial R}{\partial \dot{r}^{\alpha}}-\frac{\partial R}{\partial r^{\alpha}} & =-\mathcal{D}_{b \alpha}^{c} I^{b d} p_{c} p_{d}-\mathcal{B}_{\alpha \beta}^{c} p_{c} \dot{r}^{\beta}-\mathcal{K}_{\alpha \beta \gamma} \dot{r}^{\beta} \dot{r}^{\gamma} \\
\frac{d}{d t} p_{b} & =\mathcal{D}_{b \alpha}^{c} p_{c} \dot{r}^{\alpha}
\end{aligned}
$$

A relative equilibrium is a point $(r, \dot{r}, p)=\left(r_{0}, 0, p_{0}\right)$ which is a fixed point for the dynamics determined by equations (3.1) and (3.2). Under assumption $\mathrm{H} 1$ the point $\left(r_{0}, p_{0}\right)$ is seen to be a critical point of the amended potential. 
Because of our zero curvature assumption $\mathrm{H} 2$, the solutions of the momentum equation lie on surfaces of the form $p_{a}=P_{a}\left(r^{\alpha}, k_{b}\right), a, b=1, \ldots, m$, where $k_{b}$ are constants labeling these surfaces. Using the functions $p_{a}=$ $P_{a}\left(r^{\alpha}, k_{b}\right)$ we introduce the reduced amended potential

$$
U_{k}\left(r^{\alpha}\right)=U\left(r^{\alpha}, P_{a}\left(r^{\alpha}, k_{b}\right)\right)
$$

We think of the function $U_{k}\left(r^{\alpha}\right)$ as being the restriction of the function $U$ to the invariant manifold

$$
Q_{k}=\left\{\left(r^{\alpha}, p_{a}\right) \mid p_{a}=P_{a}\left(r^{\alpha}, k_{b}\right)\right\}
$$

Theorem 3.1 Let assumptions $H 1$ and H2 hold and let $\left(r_{0}, p_{0}\right)$, where $p_{0}=$ $P\left(r_{0}, k_{0}\right)$, be a relative equilibrium. If the reduced amended potential $U_{k_{0}}(r)$ has a nondegenerate minimum at $r_{0}$, then this equilibrium is Lyapunov stable.

Proof First, we show that the relative equilibrium

$$
r^{\alpha}=r_{0}^{\alpha}, \quad p_{a}^{0}=P_{a}\left(r_{0}^{\alpha}, k_{b}^{0}\right)
$$

of system (3.1), (3.2) is stable modulo perturbations consistent with $Q_{k_{0}}$. Consider the phase flow restricted to the invariant manifold $Q_{k_{0}}$, where $k_{0}$ corresponds to the relative equilibrium. Since $U_{k_{0}}\left(r^{\alpha}\right)$ has a nondegenerate minimum at $r_{0}^{\alpha}$, the function $\left.E\right|_{Q_{k_{0}}}$ is positive definite. By Theorem 2.6 its derivative along the flow vanishes. Using $\left.E\right|_{Q_{k_{0}}}$ as a Lyapunov function, we conclude that equations (3.1), (3.2), restricted to the manifold $Q_{k_{0}}$, have a stable equilibrium point $r_{0}^{\alpha}$ on $Q_{k_{0}}$.

To finish the proof, we need to show that equations (3.1), (3.2), restricted to nearby invariant manifolds $Q_{k}$, have stable equilibria on these manifolds.

If $k$ is sufficiently close to $k_{0}$, then by the properties of families of Morse functions (see Milnor [1963]), the function $U_{k}: Q_{k} \rightarrow \mathbb{R}$ has a nondegenerate minimum at the point $r^{\alpha}$ which is close to $r_{0}^{\alpha}$. This means that for all $k$ sufficiently close to $k_{0}$ system (3.1), (3.2) restricted to $Q_{k}$ has a stable equilibrium $r^{\alpha}$. Therefore, equilibrium (3.3) of equations (3.1), (3.2) is stable.

The stability here cannot be asymptotic, since the dynamical systems on $Q_{k}$ have a positive definite conserved quantity - the reduced energy function. 
Remark. Even though in general $P_{a}\left(r^{\alpha}, k_{b}\right)$ can not be found explicitly, the types of critical points of $U_{k}$ may be explicitly determined as follows. First of all, note that

$$
\frac{\partial p_{b}}{\partial r^{\alpha}}=\mathcal{D}_{b \alpha}^{c} p_{c}
$$

as long as $\left(r^{\alpha}, p_{a}\right) \in Q_{k}$. Therefore

$$
\frac{\partial U_{k}}{\partial r^{\alpha}}=\nabla_{\alpha} U
$$

where

$$
\nabla_{\alpha}=\frac{\partial}{\partial r^{\alpha}}+\mathcal{D}_{b \alpha}^{c} p_{c} \frac{\partial}{\partial p_{b}} .
$$

(cf. Karapetyan [1983]). The operators $\nabla_{\alpha}$ may be viewed as covariant derivatives in the vector bundle $\mathcal{D} / G \rightarrow T(Q / G)$ with fibers $\left(\mathfrak{g}^{\mathcal{D}}\right)^{*}$. They arise from the connection defined by the transport term in the momentum equation. We note that these derivatives commute in cases 1 and 2 . The relative equilibria satisfy the condition

$$
\nabla_{\alpha} U=0
$$

while the condition for stability

$$
\frac{\partial^{2} U_{k}}{\partial r^{2}} \gg 0
$$

(i.e., is positive definite) becomes the condition

$$
\nabla_{\alpha} \nabla_{\beta} U \gg 0
$$

In the commutative case this was shown by Karapetyan [1983].

Now we give the stability condition in a form similar to that of energymomentum method for holonomic systems given in Simo, Lewis, and Marsden [1991].

Theorem 3.2 (The nonholonomic energy-momentum method) Under assumptions $H 1$ and H2, the point $q_{e}=\left(r_{0}^{\alpha}, 0, p_{a}^{0}\right)$ is a relative equilibrium if and only if there is a $\xi \in \mathfrak{g}^{q_{e}}$ such that $q_{e}$ is a critical point of the augmented energy $E_{\xi}: \mathcal{D} / G \rightarrow \mathbb{R}$ (i.e., $E_{\xi}$ is a function of $(r, \dot{r}, p)$ ), defined by

$$
E_{\xi}=E-\langle p-P(r, k), \xi\rangle .
$$

This equilibrium is stable if $\delta^{2} E_{\xi}$ restricted to $T_{q_{e}} Q_{k}$ is positive definite (here $\delta$ denotes differentiation with respect to all variables except $\xi)$. 
Proof A point $q_{e} \in Q_{k}$ is a relative equilibrium if $\partial_{r^{\alpha}} U_{k}=0$. This condition is equivalent to $d\left(\left.E\right|_{Q_{k}}\right)=0$. The last equation may be represented as $d(E-\langle p-P(r, k), \xi\rangle)=0$ for some $\xi \in \mathfrak{g}^{q_{e}}$. Similarly, the condition for stability $d^{2} U_{k} \gg 0$ is equivalent to $d^{2}\left(\left.E\right|_{Q_{k}}\right) \gg 0$, which may be represented as $\left.\left(\delta^{2} E_{\xi}\right)\right|_{T_{q_{e}} Q_{k}} \gg 0$.

Note that if the momentum map is preserved, then the formula for $E_{\xi}$ becomes

$$
E_{\xi}=E-\langle p-k, \xi\rangle,
$$

which is the same as the formula for the augmented energy $E_{\xi}$ for holonomic systems.

\subsection{Examples}

There are several examples which illustrate the ideas above. For instance the falling disk, Routh's problem, and a body of revolution rolling on a horizontal plane are systems where the momentum equation defines an integrable distribution and we are left with only one shape variable. Since the stability properties of all these systems are similar, we consider here only the rolling disk. For the body of revolution on the plane see Chaplygin [1897a] and Karapetyan [1983]. For the Routh problem see Zenkov [1995].

The Rolling Disk. Consider again the disk rolling without sliding on the $x y$-plane. Recall that we have the following: Denote the coordinates of contact of the disk in the $x y$-plane by $(x, y)$. Let $\theta, \phi$, and $\psi$ denote the angle between the plane of the disk and the vertical axis, the "heading angle" of the disk, and "self-rotation" angle of the disk respectively, as was introduced earlier.

The Lagrangian and the constraints in these coordinates are given by

$$
\begin{aligned}
L= & \frac{m}{2}\left[(\xi-R(\dot{\phi} \sin \theta+\dot{\psi}))^{2}+\eta^{2} \sin ^{2} \theta+(\eta \cos \theta+R \dot{\theta})^{2}\right] \\
& +\frac{1}{2}\left[A\left(\dot{\theta}^{2}+\dot{\phi}^{2} \cos ^{2} \theta\right)+B(\dot{\phi} \sin \theta+\dot{\psi})^{2}\right]-m g R \cos \theta, \\
\dot{x}= & -\dot{\psi} R \cos \phi, \\
\dot{y}= & -\dot{\psi} R \sin \phi,
\end{aligned}
$$

where $\xi=\dot{x} \cos \phi+\dot{y} \sin \phi+R \dot{\psi}, \eta=-\dot{x} \sin \phi+\dot{y} \cos \phi$. Note that the constraints may be written as $\xi=0, \eta=0$. 
This system is invariant under the action of the group $G=S E(2) \times$ $S O(2)$; the action by the group element $(a, b, \alpha, \beta)$ is given by

$$
(\theta, \phi, \psi, x, y) \mapsto(\theta, \phi+\alpha, \psi+\beta, x \cos \alpha-y \sin \alpha+a, x \sin \alpha+y \cos \alpha+b) .
$$

Obviously,

$$
T_{q} \operatorname{Orb}(q)=\operatorname{span}\left(\frac{\partial}{\partial \phi}, \frac{\partial}{\partial \psi}, \frac{\partial}{\partial x}, \frac{\partial}{\partial y}\right)
$$

and

$$
\mathcal{D}_{q}=\operatorname{span}\left(\frac{\partial}{\partial \theta}, \frac{\partial}{\partial \phi},-R \cos \phi \frac{\partial}{\partial x}-R \sin \phi \frac{\partial}{\partial y}+\frac{\partial}{\partial \psi}\right),
$$

which imply

$$
\mathcal{S}_{q}=\mathcal{D}_{q} \cap T_{q} \operatorname{Orb}(q)=\operatorname{span}\left(\frac{\partial}{\partial \phi},-R \cos \phi \frac{\partial}{\partial x}-R \sin \phi \frac{\partial}{\partial y}+\frac{\partial}{\partial \psi}\right) .
$$

Choose vectors $(1,0,0,0),(0,1,0,0),(0,0,1,0),(0,0,0,1)$ as a basis of the Lie algebra $\mathfrak{g}$ of the group $G$. The corresponding generators are $\partial_{x}, \partial_{y},-y \partial_{x}+x \partial_{y}+\partial_{\phi}, \partial_{\psi}$. Taking into account that the generators $\partial_{\phi},-R \cos \phi \partial_{x}-R \sin \phi \partial_{y}+\partial_{\psi}$ correspond to the elements $(y,-x, 1,0)$, $(-R \cos \phi,-R \sin \phi, 0,1)$ of the Lie algebra $\mathfrak{g}$, we obtain the following momentum equations:

$$
\begin{aligned}
& \dot{p}_{1}=m R^{2} \cos \theta \dot{\theta} \dot{\psi}, \\
& \dot{p}_{2}=-m R^{2} \cos \theta \dot{\theta} \dot{\phi},
\end{aligned}
$$

where

$$
\begin{aligned}
& p_{1}=A \dot{\phi} \cos ^{2} \theta+\left(m R^{2}+B\right)(\dot{\phi} \sin \theta+\dot{\psi}) \sin \theta, \\
& p_{2}=\left(m R^{2}+B\right)(\dot{\phi} \sin \theta+\dot{\psi}),
\end{aligned}
$$

into which the constraints have been substituted. One may notice that

$$
p_{1}=\frac{\partial l_{c}}{\partial \dot{\phi}}, \quad p_{2}=\frac{\partial l_{c}}{\partial \dot{\psi}} .
$$

Solving (3.6) for $\dot{\phi}$ and $\dot{\psi}$ and substituting the solutions back in the equations (3.5) we obtain another representation of the momentum equations:

$$
\begin{aligned}
& \frac{d p_{1}}{d t}=m R^{2} \cos \theta\left(-\frac{\sin \theta}{A \cos ^{2} \theta} p_{1}+\left(\frac{1}{m R^{2}+B}+\frac{\sin ^{2} \theta}{A \cos ^{2} \theta}\right) p_{2}\right) \dot{\theta}, \\
& \frac{d p_{2}}{d t}=m R^{2} \cos \theta\left(-\frac{1}{A \cos ^{2} \theta} p_{1}+\frac{\sin \theta}{A \cos ^{2} \theta} p_{2}\right) \dot{\theta} .
\end{aligned}
$$


The right-hand sides of (3.7) do not have terms quadratic in the shape velocity $\dot{\theta}$. The distribution, defined by (3.7), is integrable and defines two integrals of the form $p_{1}=P_{1}\left(\theta, k_{1}, k_{2}\right), p_{2}=P_{2}\left(\theta, k_{1}, k_{2}\right)$. It is known that these integrals may be written down explicitly in terms of the hypergeometric function. See Appel [1900], Chaplygin [1897a], and Korteweg [1899] for details.

To carry out stability analysis, we use the remark following Theorem 3.1. Using formulae (3.6), we obtain the amended potential

$$
U(\theta, p)=\frac{1}{2}\left[\frac{\left(p_{1}-p_{2} \sin \theta\right)^{2}}{A \cos ^{2} \theta}+\frac{p_{2}^{2}}{B+m R^{2}}\right]+m g R \cos \theta .
$$

Straightforward computation shows that the condition for stability $\nabla^{2} U \gg 0$ of a relative equilibrium $\theta=\theta_{0}, p_{1}=p_{1}^{0}, p_{2}=p_{2}^{0}$ becomes

$$
\begin{aligned}
\frac{B}{A\left(m R^{2}+B\right)} & \left(p_{2}^{0}\right)^{2}+\frac{m R^{2} \cos ^{2} \theta_{0}+2 A \sin ^{2} \theta_{0}+A}{A^{2}}\left(p_{1}^{0}-p_{2}^{0} \sin \theta_{0}\right)^{2} \\
& -\frac{\left(m R^{2}+3 B\right) \sin \theta_{0}}{A\left(m R^{2}+B\right) \cos ^{2} \theta_{0}}\left(p_{1}^{0}-p_{2}^{0} \sin \theta_{0}\right) p_{2}^{0}-m g R \cos \theta_{0}>0 .
\end{aligned}
$$

Note that this condition guarantees stability here relative to $\left(\theta, \dot{\theta}, p_{1}, p_{2}\right)$; in other words we have stability modulo the action of $S E(2) \times S O(2)$.

The falling disk may be considered as a limiting case of the body of revolution which also has an integrable pure transport momentum equation (this example is treated in Chaplygin [1897a] and Karapetyan [1983]). The rolling disc has also been analyzed recently by O'Reilly [1996] and Cushman, Hermans and Kemppainen [1996]. O'Reilly considered bifurcation of relative equilibria, the stability of vertical stationary motions, as well as the possibility of sliding.

\section{The Non-Pure Transport Case}

In this section we consider the case in which the coefficients $\mathcal{D}_{\alpha \beta b}$ are not skew symmetric in $\alpha, \beta$ and the two subcases where the transport part of the momentum equation is integrable or is not integrable, respectively. In either case one may obtain asymptotic stability.

We begin by assembling some preliminary results on center manifold theory and show how they relate to the Lyapunov-Malkin theorem. The center manifold theorem provides new and useful insight into the existence of integral manifolds. These integral manifolds play a crucial role in our 
analysis. Lyapunov's original proof of the Lyapunov-Malkin theorem used a different approach to proving the existence of local integrals, as we shall discuss below. Malkin extended the result to the nonautonomous case.

\subsection{Center Manifold Theory in Stability Analysis}

Here we discuss the center manifold theory and its applications to the stability analysis of nonhyperbolic equilibria.

Consider a system of differential equations

$$
\begin{aligned}
& \dot{x}=A x+X(x, y), \\
& \dot{y}=B y+Y(x, y),
\end{aligned}
$$

where $x \in \mathbb{R}^{m}, y \in \mathbb{R}^{n}$, and $A$ and $B$ are constant matrices. It is supposed that all eigenvalues of $A$ have nonzero real parts, and all eigenvalues of $B$ have zero real parts. The functions $X, Y$ are smooth, and satisfy the conditions $X(0,0)=0, d X(0,0)=0, Y(0,0)=0, d Y(0,0)=0$. We now recall the following definition:

Definition 4.1 A smooth invariant manifold of the form $x=h(y)$ where $h$ satisfies $h(0)=0$ and $d h(0)=0$ is called a center manifold.

We are going to use the following version of the center manifold theorem following the exposition of Carr [1981] (see also Chow and Hale [1982]).

Theorem 4.2 (The center manifold theorem) If the functions $X(x, y)$, $Y(x, y)$ are $C^{k}, k \geq 2$, then there exists a (local) center manifold for (4.1), (4.2), $x=h(y),|y|<\delta$, where $h$ is $C^{k}$. The flow on the center manifold is governed by the system

$$
\dot{y}=B y+Y(h(y), y) .
$$

The next theorem explains that the reduced equation (4.3) contains information about stability of the zero solution of (4.1), (4.2).

Theorem 4.3 Suppose that the zero solution of (4.3) is stable (asymptotically stable) (unstable) and that the eigenvalues of $A$ are in the left half plane. Then the zero solution of (4.1), (4.2) is stable (asymptotically stable) (unstable). 
Let us now look at the special case of (4.2) in which the matrix $B$ vanishes. Equations (4.1), (4.2) become

$$
\begin{aligned}
& \dot{x}=A x+X(x, y), \\
& \dot{y}=Y(x, y) .
\end{aligned}
$$

Theorem 4.4 Consider the system of equations (4.4), (4.5). If $X(0, y)=0$, $Y(0, y)=0$, and all eigenvalues of the matrix A have negative real parts, then system (4.4), (4.5) has $n$ local integrals in the neighborhood of $x=0, y=0$.

Proof The center manifold in this case is given by $x=0$. Each point of the center manifold is an equilibrium of system (4.4), (4.5). For each equilibrium point $(0, y)$ of our system, consider a stable manifold $S^{s}(y)$. The center manifold and these manifolds $S^{S}(y)$ can be used for a (local) substitution $(x, y) \rightarrow(\bar{x}, \bar{y})$ such that in the new coordinates the system of differential equations become

$$
\dot{\bar{x}}=\bar{A} \bar{x}+\bar{X}(\bar{x}, \bar{y}), \quad \dot{\bar{y}}=0 .
$$

The last system of equations has $n$ integrals $\bar{y}=$ const, so that the original equation has $n$ smooth local integrals. Observe that the tangent spaces to the common level sets of these integrals at the equilibria are the planes $y=y_{0}$. Therefore, the integrals are of the form $y=f(x, k)$, where $\partial_{x} f(0, k)=0$.

The following theorem gives stability conditions for equilibria of system (4.4), (4.5).

Theorem 4.5 (Lyapunov-Malkin) Consider the system of differential equations (4.4), (4.5), where $x \in \mathbb{R}^{m}, y \in \mathbb{R}^{n}, A$ is an $m \times m$-matrix, and $X(x, y), Y(x, y)$ represent nonlinear terms. If all eigenvalues of the matrix $A$ have negative real parts, and $X(x, y), Y(x, y)$ vanish when $x=0$, then the solution $x=0, y=0$ of system (4.4), (4.5) is stable with respect to $(x, y)$, and asymptotically stable with respect to $x$. If a solution $(x(t), y(t))$ of (4.4), (4.5) is close enough to the solution $x=0, y=0$, then

$$
\lim _{t \rightarrow \infty} x(t)=0, \quad \lim _{t \rightarrow \infty} y(t)=c .
$$

The proof of this theorem consists of two steps. The first step is a reduction of the system to the common level set of integrals described in Theorem 4.4. The second step is the construction of a Lyapunov function 
for the reduced system. The details of the proof may be found in Lyapunov [1992] and Malkin [1938].

Historical Note. The proof of the Lyapunov-Malkin theorem uses the fact that the system of differential equations has local integrals, as discussed in Theorem 4.4. To prove existence of these integrals, Lyapunov uses a theorem of his own about the existence of solutions of PDE's. He does this assuming that the nonlinear terms on the right-hand sides are series in $x$ and $y$ with time-dependent bounded coefficients. Malkin generalizes Lyapunov's result for systems for which the matrix $A$ is time-dependent. We consider the nonanalytic case, and to prove existence of these local integrals, we use center manifold theory. This simplifies the arguments to some extent as well as showing how the results are related.

The following lemma specifies a class of systems of differential equations, that satisfies the conditions of the Lyapunov-Malkin theorem.

Lemma 4.6 Consider a system of differential equations of the form

$$
\dot{u}=A u+B y+\mathcal{U}(u, y), \quad \dot{y}=\mathcal{Y}(u, y),
$$

where $u \in \mathbb{R}^{m}, y \in \mathbb{R}^{n}$, $\operatorname{det} A \neq 0$, and where $\mathcal{U}$ and $\mathcal{Y}$ represent higher order nonlinear terms. There is a change of variables of the form $u=x+\phi(y)$ such that

(i) in the new variables $(x, y)$ system (4.6) becomes

$$
\dot{x}=A x+X(x, y), \quad \dot{y}=Y(x, y),
$$

(ii) if $Y(0, y)=0$, then $X(0, y)=0$ as well.

Proof Put $u=x+\phi(y)$, where $\phi(y)$ is defined by

$$
A \phi(y)+B y+\mathcal{U}(\phi(y), y)=0 \text {. }
$$

System (4.6) in the variables $(x, y)$ becomes

$$
\dot{x}=A x+X(x, y), \quad \dot{y}=Y(x, y),
$$

where

$$
\begin{aligned}
& X(x, y)=A \phi(y)+B y+\mathcal{U}(x+\phi(y), y)-\frac{\partial \phi}{\partial y} Y(x, y) \\
& Y(x, y)=\mathcal{Y}(x+\phi(y), y)
\end{aligned}
$$

Note that $Y(0, y)=0$ implies $X(0, y)=0$. 


\subsection{The Mathematical Example}

The Lyapunov-Malkin conditions. Recall from $\S 1.4$ that the equations of motion are

$$
\begin{aligned}
& \ddot{r}=-\frac{\partial V}{\partial r}-\frac{\partial b}{\partial r}(a(r) \dot{r}+b(r) p) p, \\
& \dot{p}=\frac{\partial b}{\partial r}(a(r) \dot{r}+b(r) p) \dot{r}
\end{aligned}
$$

here and below we write $r$ instead of $r^{1}$.

Recall also that a point $r=r_{0}, p=p_{0}$ is a relative equilibrium if $r_{0}$ and $p_{0}$ satisfy the condition

$$
\frac{\partial V}{\partial r}\left(r_{0}\right)+\frac{\partial b}{\partial r} b\left(r_{0}\right) p_{0}^{2}=0 .
$$

Introduce coordinates $\left(u_{1}, u_{2}, v\right)$ in the neighborhood of this equilibrium by

$$
r=r_{0}+u_{1}, \quad \dot{r}=u_{2}, \quad p=p_{0}+v .
$$

The linearized equations of motion are

$$
\begin{aligned}
\dot{u}_{1} & =u_{2}, \\
\dot{u}_{2} & =\mathcal{A} u_{2}+\mathcal{B} u_{1}+\mathcal{C} v, \\
\dot{v} & =\mathcal{D} u_{2}
\end{aligned}
$$

where

$$
\begin{aligned}
\mathcal{A} & =-\frac{\partial b}{\partial r} a p_{0} \\
\mathcal{B} & =-\frac{\partial^{2} V}{\partial r^{2}}-\left[\frac{\partial^{2} b}{\partial r^{2}} b+\left(\frac{\partial b}{\partial r}\right)^{2}\right] p_{0}^{2}, \\
\mathcal{C} & =-2 \frac{\partial b}{\partial r} b p_{0} \\
\mathcal{D} & =\frac{\partial b}{\partial r} b p_{0}
\end{aligned}
$$

and where $V, a, b$, and their derivatives are evaluated at $r_{0}$. The characteristic polynomial of these linearized equations is calculated to be

$$
\lambda\left[\lambda^{2}-\mathcal{A} \lambda-(\mathcal{B}+\mathcal{C D})\right] .
$$

It obviously has one zero root. The two others have negative real parts if

$$
\mathcal{B}+\mathcal{C D}<0, \quad \mathcal{A}<0 .
$$


These conditions imply linear stability. We discuss the meaning of these conditions later.

Next, we make the substitution $v=y+\mathcal{D} u_{1}$, which defines the new variable $y$. The (nonlinear) equations of motion become

$$
\begin{aligned}
\dot{u}_{1} & =u_{2}, \\
\dot{u}_{2} & =\mathcal{A} u_{2}+(\mathcal{B}+\mathcal{C D}) u_{1}+\mathcal{C} y+\mathcal{U}(u, y), \\
\dot{y} & =\mathcal{Y}(u, y),
\end{aligned}
$$

where $\mathcal{U}(u, y), \mathcal{Y}(u, y)$ stand for nonlinear terms, and $\mathcal{Y}(u, y)$ vanishes when $u=0$. By Lemma 4.6 there exists a further substitution $u=x+\phi(y)$ such that the equations of motion in coordinates $(x, y)$ become

$$
\begin{aligned}
& \dot{x}=P x+X(x, y), \\
& \dot{y}=Y(x, y),
\end{aligned}
$$

where $X(x, y)$ and $Y(x, y)$ satisfy the conditions $X(0, y)=0, Y(0, y)=0$. Here,

$$
P=\left(\begin{array}{cc}
0 & 1 \\
\mathcal{B}+\mathcal{C D} & \mathcal{A}
\end{array}\right) .
$$

This form enables us to use the Lyapunov-Malkin theorem and conclude that the linear stability implies nonlinear stability and in addition that we have asymptotic stability with respect to $\left(x_{1}, x_{2}\right)$.

The Energy-Momentum Method. To find a Lyapunov function based approach for analyzing the stability of the mathematical example, we introduce a modified dynamical system and use its energy function and momentum to construct a Lyapunov function for the original system. This modified system is introduced for the purpose of finding the Lyapunov function and is not used in the stability proof. We will generalize this approach below and this example may be viewed as motivation for the general approach.

Consider then the new system obtained from the Lagrangian (1.4) and the constraint $(1.5)$ by setting $a(r)=0$. Notice that $L_{c}$ stays the same and therefore, the equation of motion may be obtained from (4.7):

$$
\ddot{r}=-\frac{\partial V}{\partial r}-\frac{\partial b}{\partial r} b(r) p^{2}, \quad \dot{p}=\frac{\partial b}{\partial r} b(r) p \dot{r} .
$$

The condition for existence of the relative equilibria also stays the same. However, a crucial observation is that for the new system, the momentum equation is now integrable, in fact explicitly, so that in this example:

$$
p=k \exp \left(b^{2}(r) / 2\right) \text {. }
$$


Thus, we may proceed and use this invariant surface to perform reduction. The amended potential, defined by $U(r, p)=V(r)+\frac{1}{2} p^{2}$, becomes

$$
U_{k}(r)=V(r)+\frac{1}{2}\left(k \exp \left(b^{2}(r) / 2\right)\right)^{2} .
$$

Consider the function

$$
W_{k}=\frac{1}{2} \dot{r}^{2}+U_{k}(r)+\epsilon\left(r-r_{0}\right) \dot{r} .
$$

If $\epsilon$ is small enough and $U_{k}$ has a nondegenerate minimum, then so does $W_{k}$. Suppose that all eigenvalues of the matrix $P$ have negative real parts. Then by Theorem 4.4 equations (4.7) have a local integral $p=\mathcal{P}(r, \dot{r}, c)$. Differentiate $W_{k}$ along the vector field determined by (4.7). We obtain

$$
\begin{array}{r}
\dot{W}_{k}=-\epsilon\left(\frac{\partial^{2} V}{\partial r^{2}}\left(r_{0}\right)+\left(\frac{\partial^{2} b}{\partial r^{2}} b\left(r_{0}\right)+2\left(\frac{\partial b}{\partial r} b\left(r_{0}\right)\right)^{2}+\left(\frac{\partial b}{\partial r}\left(r_{0}\right)\right)^{2}\right) p_{0}^{2}\right) \\
-\frac{\partial b}{\partial r} a\left(r_{0}\right) p_{0} \dot{r}^{2}+\epsilon \dot{r}^{2}+\{\text { higher order terms }\} .
\end{array}
$$

Therefore, $W_{k}$ is a Lyapunov function for the flow restricted to the local invariant manifold $p=\mathcal{P}(r, \dot{r}, c)$ if

$$
\frac{\partial^{2} V}{\partial r^{2}}\left(r_{0}\right)+\left(\frac{\partial^{2} b}{\partial r^{2}} b\left(r_{0}\right)+2\left(\frac{\partial b}{\partial r} b\left(r_{0}\right)\right)^{2}+\left(\frac{\partial b}{\partial r}\left(r_{0}\right)\right)^{2}\right) p_{0}^{2}>0
$$

and

$$
\frac{\partial b}{\partial r} a\left(r_{0}\right) p_{0} \dot{r}^{2}>0
$$

Notice that the Lyapunov conditions (4.9) and (4.10) are the same as conditions (4.8).

Introduce the operator

$$
\nabla=\frac{\partial}{\partial r}+\frac{\partial b}{\partial r} b(r) p \frac{\partial}{\partial p}
$$

Then condition (4.9) may be represented as

$$
\nabla^{2} U>0
$$

which is the same as the condition for stability of stationary motions of a nonholonomic system with an integrable momentum equation (recall that 
this means that there are no terms quadratic in $\dot{r}$, only transport terms defining an integrable distribution). The left-hand side of formula (4.10) may be viewed as a derivative of the energy function

$$
E=\frac{1}{2} \dot{r}^{2}+\frac{1}{2} p^{2}+V
$$

along the flow

$$
\ddot{r}=-\frac{\partial V}{\partial r}-\frac{\partial b}{\partial r}(a(r) \dot{r}+b(r) p) p, \quad \dot{p}=\frac{\partial b}{\partial r} b(r) p \dot{r},
$$

or as a derivative of the amended potential $U$ along the vector field defined by the nontransport terms of the momentum equations

$$
\dot{p}=\frac{\partial b}{\partial r} a(r) \dot{r}^{2} .
$$

\subsection{The Nonholonomic Energy-Momentum Method}

We now generalize the energy-momentum method discussed above for the mathematical example to the general case in which the transport part of the momentum equation is integrable.

Here we assume hypothesis $\mathrm{H} 2$ in the present context, namely:

$\mathrm{H} 2$ The curvature of the connection form associated with the transport part of the momentum equation, namely $d p_{b}-\mathcal{D}_{b \alpha}^{c} p_{c} d r^{\alpha}$, is zero.

The momentum equation in this situation is

$$
\frac{d}{d t} p_{b}=\mathcal{D}_{b \alpha}^{c} p_{c} \dot{r}^{\alpha}+\mathcal{D}_{\alpha \beta b} \dot{r}^{\alpha} \dot{r}^{\beta}
$$

Hypothesis H2 implies that the form due to the transport part of the momentum equation defines an integrable distribution. Associated to this distribution, there is a family of integral manifolds

$$
p_{a}=P_{a}\left(r^{\alpha}, k_{b}\right)
$$

with $P_{a}$ satisfying the equation $d P_{b}=\mathcal{D}_{b \alpha}^{c} P_{c} d r^{\alpha}$. Note that these manifolds are not invariant manifolds of the full system under consideration because the momentum equation has non-transport terms. Substituting the functions $P_{a}\left(r^{\alpha}, k_{b}\right), k_{b}=$ const, into $E(r, \dot{r}, p)$, we obtain a function

$$
V_{k}\left(r^{\alpha}, \dot{r}^{\alpha}\right)=E\left(r^{\alpha}, \dot{r}^{\alpha}, P_{a}\left(r^{\alpha}, k_{b}\right)\right),
$$


that depends only on $\left(r^{\alpha}, \dot{r}^{\alpha}\right)$ and parametrically on $k$. This function will not be our final Lyapunov function but will be used to construct one in the proof to follow.

Pick a relative equilibrium $r^{\alpha}=r_{0}^{\alpha}, p_{a}=p_{a}^{0}$. In this context we introduce the following definiteness assumptions:

H3 At the equilibrium $r^{\alpha}=r_{0}^{\alpha}, p_{a}=p_{a}^{0}$ the two symmetric matrices $\nabla_{\alpha} \nabla_{\beta} U$ and $\left(\mathcal{D}_{\alpha \beta b}+\mathcal{D}_{\beta \alpha b}\right) I^{b c} p_{c}$ are positive definite.

Theorem 4.7 Under assumptions H2 and H3, the equilibrium $r^{\alpha}=r_{0}^{\alpha}$, $p_{a}=p_{a}^{0}$ is Lyapunov stable. Moreover, the system has local invariant manifolds that are tangent to the family of manifolds defined by the integrable transport part of the momentum equation at the relative equilibria. The relative equilibria, that are close enough to $\left(r_{0}, p_{0}\right)$, are asymptotically stable in the directions defined by these invariant manifolds. In addition, for initial conditions close enough to the equilibrium $r^{\alpha}=r_{0}^{\alpha}, p_{a}=p_{a}^{0}$, the perturbed solution approaches a nearby equilibrium.

Proof The substitution $p_{a}=p_{a}^{0}+y_{a}+\mathcal{D}_{a \alpha}^{b}\left(r_{0}\right) p_{b}^{0} u^{\alpha}$, where $u^{\alpha}=r^{\alpha}-r_{0}^{\alpha}$, eliminates the linear terms in the momentum equation. In fact, with this substitution, the equations of motion (2.8), (2.9) become

$$
\begin{aligned}
\frac{d}{d t} \frac{\partial R}{\partial \dot{r}^{\alpha}}-\frac{\partial R}{\partial r^{\alpha}}= & -\mathcal{D}_{b \alpha}^{c} I^{b d} p_{c} p_{d}-\mathcal{B}_{\alpha \beta}^{c} p_{c} \dot{r}^{\beta} \\
& -\mathcal{D}_{\beta \alpha b} I^{b c} p_{c} \dot{r}^{\beta}-\mathcal{K}_{\alpha \beta \gamma} \dot{r}^{\beta} \dot{r}^{\gamma} \\
\frac{d}{d t} y_{b}= & \mathcal{D}_{b \alpha}^{c} y_{c} \dot{r}^{\alpha}+\left(\mathcal{D}_{b \alpha}^{c}-\mathcal{D}_{b \alpha}^{c}\left(r_{0}\right)\right) p_{c}^{0} \dot{r}^{\alpha}+\mathcal{D}_{\alpha \beta b} \dot{r}^{\alpha} \dot{r}^{\beta}
\end{aligned}
$$

We will show in $\S 4.6$ that H3 implies the hypotheses of Theorem 4.4. Thus, the above equations have local integrals $y_{a}=f_{a}(r, \dot{r}, c)$, where the functions $f_{a}$ are such that $\partial_{r} f_{a}=\partial_{\dot{r}} f_{a}=0$ at the equilibria. Therefore, the original equations (2.8), (2.9) have $n$ local integrals

$$
p_{a}=\mathcal{P}_{a}\left(r^{\alpha}, \dot{r}^{\alpha}, c_{b}\right), \quad c_{b}=\text { const }
$$

where $\mathcal{P}_{a}$ are such that

$$
\frac{\partial \mathcal{P}}{\partial r^{\alpha}}=\frac{\partial P}{\partial r^{\alpha}}, \quad \frac{\partial \mathcal{P}}{\partial \dot{r}^{\alpha}}=0
$$

at the relative equilibria.

We now use the $V_{k}\left(r^{\alpha}, \dot{r}^{\alpha}\right)$ to construct a Lyapunov function to determine the conditions for asymptotic stability of the relative equilibrium $r^{\alpha}=r_{0}^{\alpha}$, 
$p_{a}=p_{a}^{0}$. We will do this in a fashion similar to that used by Chetaev [1959] and Bloch, Krishnaprasad, Marsden, and Ratiu [1994]. Without loss of generality, suppose that $g_{\alpha \beta}\left(r_{0}\right)=\delta_{\alpha \beta}$. Introduce the function

$$
W_{k}=V_{k}+\epsilon \sum_{\alpha=1}^{\sigma} u^{\alpha} \dot{r}^{\alpha} .
$$

Consider the following two manifolds at the equilibrium $\left(r_{0}^{\alpha}, 0, p_{a}^{0}\right)$ : the integral manifold of the transport equation

$$
Q_{k_{0}}=\left\{p_{a}=P_{a}\left(r^{\alpha}, k_{0}\right)\right\}
$$

and the local invariant manifold

$$
\mathcal{Q}_{c^{0}}=\left\{p_{a}=\mathcal{P}_{a}\left(r^{\alpha}, \dot{r}^{\alpha}, c^{0}\right)\right\} .
$$

Restrict the flow to the manifold $\mathcal{Q}_{c^{0}}$. Choose $\left(r^{\alpha}, \dot{r}^{\alpha}\right)$ as local coordinates on $\mathcal{Q}_{c^{0}}$, then $V_{k_{0}}$ and $W_{k_{0}}$ are functions defined on $\mathcal{Q}_{c^{0}}$. Since

$$
\frac{\partial U_{k_{0}}}{\partial r^{\alpha}}\left(r_{0}\right)=\nabla_{\alpha} U\left(r_{0}, p_{0}\right)=0
$$

and

$$
\frac{\partial^{2} U_{k_{0}}}{\partial r^{\alpha} \partial r^{\beta}}\left(r_{0}\right)=\nabla_{\alpha} \nabla_{\beta} U\left(r_{0}, p_{0}\right) \gg 0,
$$

the function $V_{k_{0}}$ is positive definite in some neighborhood of the relative equilibrium $\left(r_{0}^{\alpha}, 0\right) \in \mathcal{Q}_{c^{0}}$. The same is valid for the function $W_{k_{0}}$ if $\epsilon$ is small enough.

Now we show that $\dot{W}_{k_{0}}$ (as a function on $\mathcal{Q}_{c^{0}}$ ) is negative definite. Calculate the derivative of $W_{k_{0}}$ along the flow:

$$
\begin{aligned}
\dot{W}_{k_{0}}=g_{\alpha \beta} \dot{r}^{\alpha} \ddot{r}^{\beta} & +\frac{1}{2} \dot{g}_{\alpha \beta} \dot{r}^{\alpha} \dot{r}^{\beta}+I^{a b} P_{a} \dot{P}_{b} \\
& +\frac{1}{2} \dot{I}^{a b} P_{a} P_{b}+\dot{V}+\epsilon \sum_{\alpha=1}^{\sigma}\left(\left(\dot{r}^{\alpha}\right)^{2}+u^{\alpha} \ddot{r}^{\alpha}\right) .
\end{aligned}
$$

Using the explicit representation of equation (2.8), we obtain

$$
\begin{array}{r}
g_{\alpha \beta} \ddot{r}^{\beta}+\dot{g}_{\alpha \beta} \dot{r}^{\beta}=\frac{1}{2} \frac{\partial g_{\beta \gamma}}{\partial r^{\alpha}} \dot{r}^{\beta} \dot{r}^{\gamma}-\frac{\partial V}{\partial r^{\alpha}}-\frac{1}{2} \frac{\partial I^{a b}}{\partial r^{\alpha}} \mathcal{P}_{a} \mathcal{P}_{b}-\mathcal{D}_{b \alpha}^{c} I^{b d} \mathcal{P}_{c} \mathcal{P}_{d} \\
-\mathcal{D}_{\beta \alpha b} I^{b c} \mathcal{P}_{c} \dot{r}^{\beta}-\mathcal{B}_{\alpha \beta}^{c} \mathcal{P}_{c} \dot{r}^{\beta}-\mathcal{K}_{\alpha \beta \gamma} \dot{r}^{\beta} \dot{r}^{\gamma}
\end{array}
$$


Therefore,

$$
\begin{aligned}
g_{\alpha \beta} \dot{r}^{\alpha} \ddot{r}^{\beta}+ & \frac{1}{2} \dot{g}_{\alpha \beta} \dot{r}^{\alpha} \dot{r}^{\beta}+I^{a b} P_{a} \dot{P}_{b}+\frac{1}{2} \dot{I}^{a b} P_{a} P_{b}+\dot{V} \\
= & -\frac{1}{2} \dot{g}_{\alpha \beta} \dot{r}^{\alpha} \dot{r}^{\beta}+\frac{1}{2} \frac{\partial g_{\beta \gamma}}{\partial r^{\alpha}} \dot{r}^{\alpha} \dot{r}^{\beta} \dot{r}^{\gamma}-\frac{\partial V}{\partial r^{\alpha}} \dot{r}^{\alpha}-\frac{1}{2} \frac{\partial I^{a b}}{\partial r^{\alpha}} \mathcal{P}_{a} \mathcal{P}_{b} \dot{r}^{\alpha} \\
& -\mathcal{D}_{b \alpha}^{c} I^{b d} \mathcal{P}_{c} \mathcal{P}_{d} \dot{r}^{\alpha}-\mathcal{D}_{\beta \alpha b} I^{b c} \mathcal{P}_{c} \dot{r}^{\alpha} \dot{r}^{\beta}-\mathcal{B}_{\alpha \beta}^{c} \mathcal{P}_{c} \dot{r}^{\alpha} \dot{r}^{\beta} \\
& -\mathcal{K}_{\alpha \beta \gamma} \dot{r}^{\alpha} \dot{r}^{\beta} \dot{r}^{\gamma}+I^{a b} \mathcal{D}_{b \alpha}^{c} P_{a} P_{c} \dot{r}^{\alpha}+\frac{1}{2} \frac{\partial I^{a b}}{\partial r^{\alpha}} P_{a} P_{b} \dot{r}^{\alpha}+\dot{V} .
\end{aligned}
$$

Using skew-symmetry of $\mathcal{B}_{\alpha \beta}^{c}$ and $\mathcal{K}_{\alpha \beta \gamma}$ with respect to $\alpha, \beta$ and canceling the terms

$$
-\frac{1}{2} \dot{g}_{\alpha \beta} \dot{r}^{\alpha} \dot{r}^{\beta}+\frac{1}{2} \frac{\partial g_{\beta \gamma}}{\partial r^{\alpha}} \dot{r}^{\alpha} \dot{r}^{\beta} \dot{r}^{\gamma}-\frac{\partial V}{\partial r^{\alpha}} \dot{r}^{\alpha}+\dot{V}
$$

we obtain

$$
\begin{aligned}
g_{\alpha \beta} \dot{r}^{\alpha} & \ddot{r}^{\beta}+\frac{1}{2} \dot{g}_{\alpha \beta} \dot{r}^{\alpha} \dot{r}^{\beta}+I^{a b} P_{a} \dot{P}_{b}+\frac{1}{2} \dot{I}^{a b} P_{a} P_{b}+\dot{V} \\
& =-\mathcal{D}_{\beta \alpha b} I^{b c} \mathcal{P}_{c} \dot{r}^{\alpha} \dot{r}^{\beta}+\left(\frac{1}{2} \frac{\partial I^{a b}}{\partial r^{\alpha}}+I^{a c} \mathcal{D}_{c \alpha}^{b}\right)\left(P_{a} P_{b}-\mathcal{P}_{a} \mathcal{P}_{b}\right) \dot{r}^{\alpha}
\end{aligned}
$$

Substituting (4.14) in (4.12) and determining $\ddot{r}^{\alpha}$ from (4.13), we obtain

$$
\begin{aligned}
\dot{W}_{k_{0}}= & -\mathcal{D}_{\beta \alpha b} I^{b c} \mathcal{P}_{c} \dot{r}^{\alpha} \dot{r}^{\beta}+\epsilon \sum_{\alpha=1}^{\sigma}\left(\dot{r}^{\alpha}\right)^{2} \\
& -\epsilon \sum_{\gamma=1}^{\sigma} g^{\alpha \beta} u^{\gamma}\left(\frac{\partial V}{\partial r^{\alpha}}+\frac{1}{2} \frac{\partial I^{a b}}{\partial r^{\alpha}} \mathcal{P}_{a} \mathcal{P}_{b}+\mathcal{D}_{b \alpha}^{c} I^{b d} \mathcal{P}_{c} \mathcal{P}_{d}\right) \\
& +\epsilon \sum_{\gamma=1}^{\sigma} g^{\alpha \gamma} u^{\gamma}\left(-\dot{g}_{\alpha \beta} \dot{r}^{\beta}+\frac{1}{2} \frac{\partial g_{\beta \gamma}}{\partial r^{\alpha}} \dot{r}^{\beta} \dot{r}^{\gamma}-\mathcal{B}_{\alpha \beta}^{c} \mathcal{P}_{c} \dot{r}^{\beta}\right. \\
& \left.-\mathcal{D}_{\beta \alpha b} I^{b c} \mathcal{P}_{c} \dot{r}^{\beta}-\mathcal{K}_{\alpha \beta \gamma} \dot{r}^{\beta} \dot{r}^{\gamma}\right) \\
& +\frac{1}{2} \frac{\partial I^{a b}}{\partial r^{\alpha}}\left(P_{a} P_{b}-\mathcal{P}_{a} \mathcal{P}_{b}\right) \dot{r}^{\alpha}+I^{a c} \mathcal{D}_{c \alpha}^{b}\left(P_{a} P_{b}-\mathcal{P}_{a} \mathcal{P}_{b}\right) \dot{r}^{\alpha}
\end{aligned}
$$

Since

$$
\frac{\partial V}{\partial r^{\alpha}}+\frac{1}{2} \frac{\partial I^{a b}}{\partial r^{\alpha}} \mathcal{P}_{a} \mathcal{P}_{b}+\mathcal{D}_{b \alpha}^{c} I^{b d} \mathcal{P}_{c} \mathcal{P}_{d}=0
$$

at the equilibrium and the linear terms in the Taylor expansions of $\mathcal{P}$ and $P$ are the same,

$$
\frac{\partial V}{\partial r^{\alpha}}+\frac{1}{2} \frac{\partial I^{a b}}{\partial r^{\alpha}} \mathcal{P}_{a} \mathcal{P}_{b}+\mathcal{D}_{b \alpha}^{c} I^{b d} \mathcal{P}_{c} \mathcal{P}_{d}=F_{\alpha \beta} u^{\beta}+\{\text { nonlinear terms }\}
$$


where

$$
\begin{aligned}
F_{\alpha \beta}= & \frac{\partial}{\partial r^{\beta}}\left(\frac{\partial V}{\partial r^{\alpha}}+\frac{1}{2} \frac{\partial I^{a b}}{\partial r^{\alpha}} P_{a} P_{b}+\mathcal{D}_{b \alpha}^{c} I^{b d} P_{c} P_{d}\right) \\
= & \frac{\partial^{2} V}{\partial r^{\alpha} \partial r^{\beta}}+\frac{1}{2} \frac{\partial^{2} I^{a b}}{\partial r^{\alpha} \partial r^{\beta}} P_{a} P_{b}+\frac{\partial I^{a b}}{\partial r^{\alpha}} P_{a} \frac{\partial P_{b}}{\partial r^{\beta}} \\
& +\frac{\partial}{\partial r^{\beta}}\left(\mathcal{D}_{b \alpha}^{c} I^{b d}\right) P_{c} P_{d}+\mathcal{D}_{b \alpha}^{c} I^{b d}\left(\frac{\partial P_{c}}{\partial r^{\beta}} P_{d}+P_{c} \frac{\partial P_{d}}{\partial r^{\beta}}\right) \\
= & \frac{\partial^{2} V}{\partial r^{\alpha} \partial r^{\beta}}+\frac{1}{2} \frac{\partial^{2} I^{a b}}{\partial r^{\alpha} \partial r^{\beta}} P_{a} P_{b}+\frac{\partial I^{a b}}{\partial r^{\alpha}} P_{a} \mathcal{D}_{b \beta}^{c} P_{c} \\
& +\frac{\partial}{\partial r^{\beta}}\left(\mathcal{D}_{b \alpha}^{c} I^{b d}\right) P_{c} P_{d}+\mathcal{D}_{b \alpha}^{c} I^{b d}\left(\mathcal{D}_{c \beta}^{a} P_{a} P_{d}+\mathcal{D}_{d \beta}^{a} P_{a} P_{c}\right) \\
= & \nabla_{\alpha} \nabla_{\beta} U .
\end{aligned}
$$

In the last formula all the terms are evaluated at the equilibrium.

Taking into account that $g_{\alpha \beta}=\delta_{\alpha \beta}+O(u)$, that the Taylor expansion of $P_{a} P_{b}-\mathcal{P}_{a} \mathcal{P}_{b}$ starts from the terms of the second order, and using (4.16), we obtain from (4.15)

$$
\begin{aligned}
\dot{W}_{k_{0}}= & -\mathcal{D}_{\beta \alpha b} I^{b c}\left(r_{0}\right) p_{c}^{0} \dot{r}^{\alpha} \dot{r}^{\beta}-\epsilon F_{\alpha \beta} u^{\alpha} u^{\beta}+\epsilon \sum_{\alpha=1}^{\sigma}\left(\dot{r}^{\alpha}\right)^{2} \\
& -\epsilon\left(\mathcal{D}_{\beta \alpha b} I^{b c}\left(r_{0}\right) p_{c}^{0}+\mathcal{B}_{\alpha \beta}^{c}\left(r_{0}\right) p_{c}^{0}\right) u^{\alpha} \dot{r}^{\beta}+\{\text { cubic terms }\} .
\end{aligned}
$$

Therefore, the condition $\left(\mathcal{D}_{\alpha \beta b}+\mathcal{D}_{\beta \alpha b}\right) I^{b c} p_{c}^{0} \gg 0$ implies that $\dot{W}_{k_{0}}$ is negative definite if $\epsilon$ is small enough and positive. Thus, $W_{k_{0}}$ is a Lyapunov function for the flow on $\mathcal{Q}_{c^{0}}$, and therefore the equilibrium $\left(r_{0}^{\alpha}, 0\right)$ for the flow on $\mathcal{Q}_{c^{0}}$ is asymptotically stable.

Using the same arguments we used in the proof of Theorem 3.1, we conclude that the equilibria on the nearby invariant manifolds $\mathcal{Q}_{k}$ are asymptotically stable as well.

There is an alternative way to state the above theorem, which uses the basic intuition we used to find the Lyapunov function.

Theorem 4.8 (The nonholonomic energy-momentum method) Under the assumption that H2 holds, the point $q_{e}=\left(r_{0}^{\alpha}, 0, p_{a}^{0}\right)$ is a relative equilibrium if and only if there is a $\xi \in \mathfrak{g}^{q_{e}}$ such that $q_{e}$ is a critical point of the augmented energy $E_{\xi}=E-\langle p-P(r, k), \xi\rangle$. Assume that

(i) $\delta^{2} E_{\xi}$ restricted to $T_{q_{e}} Q_{k}$ is positive definite (here $\delta$ denotes differentiation by all variables except $\xi$ ); 
(ii) the quadratic form defined by the flow derivative of the augmented energy is negative definite at $q_{e}$.

Then H3 holds and this equilibrium is Lyapunov stable and asymptotically stable in the directions of due to the invariant manifolds (4.11).

Proof We have already shown in Theorem 3.2 that positive definiteness of $\left.\delta^{2} E_{\xi}\right|_{T_{e} Q_{k}}$ is equivalent to the condition $\nabla_{\alpha} \nabla_{\beta} U \gg 0$. To complete the proof, we need to show that the requirement (ii) of the theorem is equivalent to the condition $\left(\mathcal{D}_{\alpha \beta b}+\mathcal{D}_{\beta \alpha b}\right) I^{b c}\left(r_{0}\right) p_{c}^{0} \gg 0$. Compute the flow derivative of $E_{\xi}$ :

$$
\dot{E}_{\xi}=\dot{E}-\langle\dot{p}-\dot{P}, \xi\rangle=\dot{E}-\left(\mathcal{D}_{a \alpha}^{b} p_{b} \dot{r}^{\alpha}+\mathcal{D}_{\alpha \beta a} \dot{r}^{\alpha} \dot{r}^{\beta}-\mathcal{D}_{a \alpha}^{b} P_{b} \dot{r}^{\alpha}\right) \xi^{a} .
$$

Since at the equilibrium $p=P, \xi^{a}=I^{a b} p_{b}$, and $\dot{E}=0$ (Theorem 2.6), we obtain

$$
\dot{E}_{\xi}=-\mathcal{D}_{\alpha \beta a} I^{a b}\left(r_{0}\right) p_{b}^{0} \dot{r}^{\alpha} \dot{r}^{\beta} .
$$

The condition $\dot{E}_{\xi} \ll 0$ is thus equivalent to $\left(\mathcal{D}_{\alpha \beta b}+\mathcal{D}_{\beta \alpha b}\right) I^{b c}\left(r_{0}\right) p_{c}^{0} \gg 0$.

For some examples, such as the roller racer, we need to consider a degenerate case of the above analysis. Namely, we consider a nongeneric case, when $U=\frac{1}{2} I^{a b}(r) p_{a} p_{b}$ (the original system has no potential energy), and the components of the locked inertia tensor $I^{a b}$ satisfy the condition

$$
\frac{1}{2} \frac{\partial I^{a b}}{\partial r^{\alpha}}+I^{a c} \mathcal{D}_{c \alpha}^{b}=0
$$

Recalling our definition of the covariant derivatives in formula (3.4), we observe that the covariant derivatives $\nabla_{\alpha} U$ of the amended potential are equal to zero, and further that the equations of motion (2.8), (2.9) become

$$
\begin{aligned}
\frac{d}{d t}\left(g_{\alpha \beta} \dot{r}^{\beta}\right)-\frac{1}{2} \frac{\partial g_{\beta \gamma}}{\partial r^{\alpha}} \dot{r}^{\beta} \dot{r}^{\gamma} & =-\mathcal{D}_{\beta \alpha b} I^{b c} p_{c} \dot{r}^{\beta}-\mathcal{B}_{\alpha \beta}^{c} p_{c} \dot{r}^{\beta}-\mathcal{K}_{\alpha \beta \gamma} \dot{r}^{\beta} \dot{r}^{\gamma} \\
\frac{d}{d t} p_{b} & =\mathcal{D}_{b \alpha}^{c} p_{c} \dot{r}^{\alpha}+\mathcal{D}_{\alpha \beta b} \dot{r}^{\alpha} \dot{r}^{\beta}
\end{aligned}
$$

Thus, we obtain an $(m+\sigma)$-dimensional manifold of equilibria $r=r_{0}, p=$ $p_{0}$ of these equations. Further, we cannot apply Theorem 4.7 because the condition $\nabla^{2} U \gg 0$ fails. However, we can do a similar type of stability analysis as follows. 
As before, set

$$
V_{k}=E(r, \dot{r}, P(r, k))=\frac{1}{2} g_{\alpha \beta} \dot{r}^{\alpha} \dot{r}^{\beta}+\frac{1}{2} I^{a b}(r) P_{a}(r, k) P_{b}(r, k) .
$$

Note that $P$ satisfies the equation

$$
\frac{\partial P_{b}}{\partial r^{\alpha}}=\mathcal{D}_{b \alpha}^{c} P_{c}
$$

which implies that

$$
\begin{aligned}
\frac{\partial}{\partial r^{\alpha}}\left(\frac{1}{2} I^{a b}(r) P_{a} P_{b}\right) & =\frac{1}{2} \frac{\partial I^{a b}}{\partial r^{\alpha}} P_{a} P_{b}+I^{a b} P_{a} \frac{\partial P_{b}}{\partial r^{\alpha}} \\
& =\left(\frac{1}{2} \frac{\partial I^{a b}}{\partial r^{\alpha}}+I^{a b} \mathcal{D}_{b \alpha}^{c}\right) P_{a} P_{b}=0 .
\end{aligned}
$$

Therefore

$$
\frac{1}{2} I^{a b} P_{a} P_{b}=\text { const }
$$

and

$$
V_{k}=\frac{1}{2} g_{\alpha \beta} \dot{r}^{\alpha} \dot{r}^{\beta}
$$

(up to an additive constant). Thus, $V_{k}$ is a positive definite function with respect to $\dot{r}$. Compute $\dot{V}_{k}$ :

$$
\dot{V}_{k}=g_{\alpha \beta} \dot{r}^{\alpha} \ddot{r}^{\beta}+\dot{g}_{\alpha \beta} \dot{r}^{\alpha} \dot{r}^{\beta}=-\mathcal{D}_{\beta \alpha b} I^{b c} p_{c} \dot{r}^{\alpha} \dot{r}^{\beta}+O\left(\dot{r}^{3}\right) .
$$

Suppose that $\left(\mathcal{D}_{\alpha \beta b}+\mathcal{D}_{\beta \alpha b}\right)\left(r_{0}\right) I^{b c}\left(r_{0}\right) p_{c}^{0} \gg 0$. Now the linearization of equations (4.18) and (4.19) about the relative equilibria given by setting $\dot{r}=0$ has $(m+\sigma)$ zero eigenvalues corresponding to the $r$ and $p$ directions. Since the matrix corresponding to $\dot{r}$-directions of the linearized system is of the form $D+G$, where $D$ is positive definite and symmetric (in fact, $\left.D=\frac{1}{2}\left(\mathcal{D}_{\alpha \beta b}+\mathcal{D}_{\beta \alpha b}\right)\left(r_{0}\right) I^{b c}\left(r_{0}\right) p_{c}^{0}\right)$ and $G$ is skew-symmetric, the determinant of $D+G$ is not equal to zero. This follows from the observation that $x^{t}(D+$ $G) x=x^{t} D x>0$ for $D$ positive-definite and $G$ skew-symmetric. Thus using Theorem 4.4, we find that the equations of motion have local integrals

$$
r=\mathcal{R}(\dot{r}, k), \quad p=\mathcal{P}(\dot{r}, k) .
$$

Therefore $V_{k}$ restricted to a common level set of these integrals is a Lyapunov function for the restricted system. Thus, an equilibrium $r=r_{0}, p=p_{0}$ is stable with respect to $(r, \dot{r}, p)$ and asymptotically stable with respect to $\dot{r}$ if

$$
\left(\mathcal{D}_{\alpha \beta b}+\mathcal{D}_{\beta \alpha b}\right) I^{b c}\left(r_{0}\right) p_{c}^{0} \gg 0 .
$$

Summarizing, we have: 
Theorem 4.9 Under assumptions H2 if $V=0$ and conditions (4.17) and (4.20) hold, the nonholonomic equations of motion have an $(m+\sigma)$-dimensional manifold of equilibria parametrized by $r$ and $p$. An equilibrium $r=r_{0}, p=p_{0}$ is stable with respect to $(r, \dot{r}, p)$ and asymptotically stable with respect to $\dot{r}$.

\subsection{The Roller Racer}

The roller racer provides an illustration of Theorem 4.9. Recall that the Lagrangian and the constraints are

$$
L=\frac{1}{2} m\left(\dot{x}^{2}+\dot{y}^{2}\right)+\frac{1}{2} I_{1} \dot{\theta}^{2}+\frac{1}{2} I_{2}(\dot{\theta}+\dot{\phi})^{2}
$$

and

$$
\begin{aligned}
& \dot{x}=\cos \theta\left(\frac{d_{1} \cos \phi+d_{2}}{\sin \phi} \dot{\theta}+\frac{d_{2}}{\sin \phi} \dot{\phi}\right), \\
& \dot{y}=\sin \theta\left(\frac{d_{1} \cos \phi+d_{2}}{\sin \phi} \dot{\theta}+\frac{d_{2}}{\sin \phi} \dot{\phi}\right) .
\end{aligned}
$$

The configuration space is $S E(2) \times S O(2)$ and, as observed earlier, the Lagrangian and the constraints are invariant under the left action of $S E(2)$ on the first factor of the configuration space.

The nonholonomic momentum is

$$
p=m\left(d_{1} \cos \phi+d_{2}\right)(\dot{x} \cos \theta+\dot{y} \sin \theta)+\left[\left(I_{1}+I_{2}\right) \dot{\theta}+I_{2} \dot{\phi}\right] \sin \phi .
$$

See Tsakiris [1995] for details of this calculation. The momentum equation is

$$
\begin{aligned}
\left.\dot{p}=\frac{\left(\left(I_{1}+I_{2}\right) \cos \phi\right.}{m\left(d_{1} \cos \phi\right.}+d_{2} d_{1}\left(d_{1} \cos \phi+d_{2}\right)\right) \sin \phi & \dot{\phi} \\
& +\frac{m\left(I_{1}+I_{2}\right) \sin ^{2} \phi}{m\left(d_{1} \cos \phi\right)\left(I_{2} d_{1} \cos \phi-d_{2}\right)^{2}+\left(I_{1}+I_{2}\right) \sin ^{2} \phi} \dot{\phi}^{2} .
\end{aligned}
$$

Rewriting the Lagrangian using $p$ instead of $\dot{\theta}$, we obtain the energy function for the roller racer:

$$
E=\frac{1}{2} g(\phi) \dot{\phi}^{2}+\frac{1}{2} I(\phi) p^{2}
$$

where

$$
g(\phi)=I_{2}+\frac{m d_{2}^{2}}{\sin ^{2} \phi}-\frac{\left[m\left(d_{1} \cos \phi+d_{2}\right) d_{2}+I_{2} \sin ^{2} \phi\right]^{2}}{\sin ^{2} \phi\left[m\left(d_{1} \cos \phi+d_{2}\right)^{2}+\left(I_{1}+I_{2}\right) \sin ^{2} \phi\right]}
$$


and

$$
I(\phi)=\frac{1}{m\left(d_{1} \cos \phi+d_{2}\right)^{2}+\left(I_{1}+I_{2}\right) \sin ^{2} \phi} .
$$

The amended potential is given by

$$
U=\frac{p^{2}}{2\left[m\left(d_{1} \cos \phi+d_{2}\right)^{2}+\left(I_{1}+I_{2}\right) \sin ^{2} \phi\right]},
$$

which follows directly from (2.7) and (4.21).

Straightforward computations show that the locked inertia tensor $I(\phi)$ satisfies condition (4.17), and thus the roller racer has a two-dimensional manifold of relative equilibria parametrized by $\phi$ and $p$. These relative equilibria are motions of the roller racer in circles about the point of intersection of lines through the axles. For such motions, $p$ is the system momentum about this point scaled by a factor of $\sin \phi$, where $\phi$ is the relative angle between the two bodies.

Therefore, we may apply the energy-momentum stability conditions (4.20) obtained in $\S 4.3$ for the degenerate case. Multiplying the coefficient of the nontransport term of the momentum equation, evaluated at $\phi_{0}$, by $I(\phi) p_{0}$ and omitting a positive factor, we obtain the condition for stability of a relative equilibrium $\phi=\phi_{0}, p=p_{0}$ of the roller racer:

$$
\left(d_{1}+d_{2} \cos \phi_{0}\right)\left(I_{2} d_{1} \cos \phi_{0}-I_{1} d_{2}\right) p_{0}>0 .
$$

Note that this equilibrium is stable modulo $S E(2)$ and in addition asymptotically stable with respect to $\dot{\phi}$.

\subsection{Nonlinear Stability by the Lyapunov-Malkin Method}

Here we study stability using the Lyapunov-Malkin approach; correspondingly, we do not a priori assume hypotheses $\mathrm{H} 1$ (skewness of $\mathcal{D}_{\alpha \beta b}$ in $\alpha, \beta$ ), $\mathrm{H} 2$ (a curvature is zero) or H3 (definiteness of second variations). Rather, at the end of this section we will make eigenvalue hypotheses.

We consider the most general case, when the connection due to the transport part of the momentum equation is not necessary flat and when the nontransport terms of the momentum equation are not equal to zero. In the case when $\mathfrak{g}^{q}$ is commutative, this analysis was done by Karapetyan [1980]. Our main goal here is to show that this method extends to the noncommutative case as well.

We start by computing the linearization of equations (2.8) and (2.9). Introduce coordinates $\left(u^{\alpha}, v^{\alpha}, w_{a}\right)$ in the neighborhood of the equilibrium 
$r=r_{0}, p=p_{0}$ by the formulae

$$
r^{\alpha}=r_{0}^{\alpha}+u^{\alpha}, \quad \dot{r}^{\alpha}=v^{\alpha}, \quad p_{a}=p_{a}^{0}+w_{a} .
$$

The linearized momentum equation is

$$
\dot{w}_{b}=\mathcal{D}_{b \alpha}^{c}\left(r_{0}\right) p_{c}^{0} v^{\alpha} .
$$

To find the linearization of (2.8), we start by rewriting its right-hand side explicitly. Since $R=\frac{1}{2} g_{\alpha \beta} \dot{r}^{\alpha} \dot{r}^{\beta}-\frac{1}{2} I^{a b} p_{a} p_{b}-V$, equation (2.8) becomes

$$
\begin{aligned}
g_{\alpha \beta} \ddot{r}^{\beta} & +\dot{g}_{\alpha \beta} \dot{r}^{\alpha} \dot{r}^{\beta}-\frac{1}{2} \frac{\partial g_{\beta \gamma}}{\partial r^{\alpha}} \dot{r}^{\beta} \dot{r}^{\gamma}+\frac{1}{2} \frac{\partial I^{a b}}{\partial r^{\alpha}} p_{a} p_{b}+\frac{\partial V}{\partial r^{\alpha}} \\
& =-\mathcal{D}_{c \alpha}^{a} I^{c d} p_{a} p_{d}-\mathcal{D}_{\beta \alpha c} I^{c a} p_{a} \dot{r}^{\beta}-\mathcal{B}_{\alpha \beta}^{a} p_{a} \dot{r}^{\beta}-\mathcal{K}_{\alpha \beta \gamma} \dot{r}^{\beta} \dot{r}^{\gamma}
\end{aligned}
$$

Keeping only the linear terms, we obtain

$$
\begin{gathered}
g_{\alpha \beta}\left(r_{0}\right) \ddot{r}^{\beta}+\frac{\partial^{2} V}{\partial r^{\alpha} \partial r^{\beta}}\left(r_{0}\right) u^{\beta}+\frac{1}{2} \frac{\partial^{2} I^{a b}}{\partial r^{\alpha} \partial r^{\beta}}\left(r_{0}\right) p_{a}^{0} p_{b}^{0} u^{\beta}+\frac{\partial I^{a b}}{\partial r^{\alpha}}\left(r_{0}\right) p_{a}^{0} w_{b} \\
=-\mathcal{D}_{c \alpha}^{a} I^{c d}\left(r_{0}\right) p_{a}^{0} w_{d}-\mathcal{D}_{c \alpha}^{a} I^{c d}\left(r_{0}\right) p_{d}^{0} w_{a}-\frac{\partial \mathcal{D}_{c \alpha}^{a} I^{c d}}{\partial r^{\beta}}\left(r_{0}\right) p_{a}^{0} p_{d}^{0} u^{\beta} \\
-\mathcal{D}_{\beta \alpha c} I^{c a}\left(r_{0}\right) p_{a}^{0} v^{\beta}-\mathcal{B}_{\alpha \beta}^{a}\left(r_{0}\right) p_{a}^{0} v^{\beta} .
\end{gathered}
$$

Next, introduce matrices $\mathcal{A}, \mathcal{B}, \mathcal{C}$, and $\mathcal{D}$ by

$$
\begin{aligned}
\mathcal{A}_{\alpha \beta} & =-\left(\mathcal{D}_{\beta \alpha c} I^{c a}\left(r_{0}\right) p_{a}^{0}+\mathcal{B}_{\alpha \beta}^{a}\left(r_{0}\right) p_{a}^{0}\right), \\
\mathcal{B}_{\alpha \beta} & =-\left(\frac{\partial^{2} V}{\partial r^{\alpha} \partial r^{\beta}}\left(r_{0}\right)+\frac{1}{2} \frac{\partial^{2} I^{a b}}{\partial r^{\alpha} \partial r^{\beta}}\left(r_{0}\right) p_{a}^{0} p_{b}^{0}+\frac{\partial \mathcal{D}_{c \alpha}^{a} I^{c b}}{\partial r^{\beta}}\left(r_{0}\right) p_{a}^{0} p_{b}^{0}\right), \\
\mathcal{C}_{\alpha}^{a} & =-\left(\frac{\partial I^{a b}}{\partial r^{\alpha}}\left(r_{0}\right) p_{b}^{0}+\mathcal{D}_{c \alpha}^{b} I^{c a}\left(r_{0}\right) p_{b}^{0}+\mathcal{D}_{c \alpha}^{a} I^{c b}\left(r_{0}\right) p_{b}^{0}\right), \\
\mathcal{D}_{a \alpha} & =\mathcal{D}_{a \alpha}^{c}\left(r_{0}\right) p_{c}^{0} .
\end{aligned}
$$

Using these notations and making a choice of $r^{\alpha}$ such that $g_{\alpha \beta}\left(r_{0}\right)=\delta_{\alpha \beta}$, we can represent the equation of motion in the form

$$
\begin{aligned}
& \dot{u}^{\alpha}=v^{\alpha}, \\
& \dot{v}^{\alpha}=\mathcal{A}_{\beta}^{\alpha} v^{\beta}+\mathcal{B}_{\beta}^{\alpha} u^{\beta}+\mathcal{C}^{\alpha a} w_{a}+\mathcal{V}^{\alpha}(u, v, w), \\
& \dot{w}_{a}=\mathcal{D}_{a \alpha} v^{\alpha}+\mathcal{W}_{a}(u, v, w),
\end{aligned}
$$


where $\mathcal{V}$ and $\mathcal{W}$ stand for nonlinear terms, and where

$$
\begin{aligned}
\mathcal{A}_{\beta}^{\alpha} & =\delta^{\alpha \gamma} \mathcal{A}_{\gamma \beta}, \\
\mathcal{B}_{\beta}^{\alpha} & =\delta^{\alpha \gamma} \mathcal{B}_{\gamma \beta}, \\
\mathfrak{C}^{\alpha a} & =\delta^{\alpha \gamma} \mathcal{C}_{\gamma}^{a} .
\end{aligned}
$$

$\left(\operatorname{Or} \mathcal{A}_{\beta}^{\alpha}=g^{\alpha \gamma} \mathcal{A}_{\gamma \beta}, \mathcal{B}_{\beta}^{\alpha}=g^{\alpha \gamma} \mathcal{B}_{\gamma \beta}, \mathcal{C}^{\alpha a}=g^{\alpha \gamma} \bigodot_{\gamma}^{a}\right.$ if $\left.g_{\alpha \beta}\left(r_{0}\right) \neq \delta_{\alpha \beta}.\right)$ Note that

$$
\mathcal{W}_{a}=\left(\mathcal{D}_{a \alpha}^{c}\left(p_{c}^{0}+w_{c}\right)-\mathcal{D}_{a \alpha}\right) v^{\alpha}+\mathcal{D}_{\alpha \beta a} v^{\alpha} v^{\beta} .
$$

The next step is to eliminate the linear terms from (4.27). Putting

$$
w_{a}=\mathcal{D}_{a \alpha} u^{\alpha}+z_{a},
$$

(4.27) becomes

$$
\dot{z}_{a}=z_{a}(u, v, z),
$$

where $z_{a}(u, v, z)$ represents nonlinear terms. Formula (4.28) leads to

$$
z_{a}(u, v, z)=z_{a \alpha}(u, v, z) v^{\alpha} .
$$

In particular, $z_{a}(u, 0, z)=0$. Equations (4.25), (4.26), (4.27) in the variables $(u, v, z)$ become

$$
\begin{aligned}
& \dot{u}^{\alpha}=v^{\alpha} \\
& \dot{v}^{\alpha}=\mathcal{A}_{\beta}^{\alpha} v^{\beta}+\left(\mathcal{B}_{\beta}^{\alpha}+\mathcal{C}^{\alpha a} \mathcal{D}_{a \beta}\right) u^{\beta}+\mathcal{C}^{\alpha a} z_{a}+\mathcal{V}^{\alpha}\left(u, v, z_{a}+\mathcal{D}_{a \alpha} u^{\alpha}\right), \\
& \dot{z}_{a}=z_{a}(u, v, w) .
\end{aligned}
$$

Using Lemma 4.6, we find a substitution $x^{\alpha}=u^{\alpha}+\phi^{\alpha}(z), y^{\alpha}=v^{\alpha}$ such that in the variables $(x, y, z)$ we obtain

$$
\begin{aligned}
& \dot{x}^{\alpha}=y^{\alpha}+X^{\alpha}(x, y, z), \\
& \dot{y}^{\alpha}=\mathcal{A}_{\beta}^{\alpha} y^{\beta}+\left(\mathcal{B}_{\beta}^{\alpha}+\mathcal{C}^{\alpha a} \mathcal{D}_{a \beta}\right) x^{\beta}+Y^{\alpha}(x, y, z), \\
& \dot{z}_{a}=Z_{a}(x, y, z),
\end{aligned}
$$

where the nonlinear terms $X(x, y, z), Y(x, y, z),, Z(x, y, z)$ vanish if $x=0$ and $y=0$. Therefore, we can apply the Lyapunov-Malkin theorem and conclude:

Theorem 4.10 The equilibrium $x=0, y=0, z=0$ of system (4.29) is stable with respect to $(x, y, z)$ and asymptotically stable with respect to $(x, y)$ if all eigenvalues of the matrix

$$
\left(\begin{array}{cc}
0 & I \\
\mathcal{B}+\mathcal{C D} & \mathcal{A}
\end{array}\right)
$$

have negative real parts. 


\subsection{The Lyapunov-Malkin and the Energy-Momentum Meth- ods}

Here we introduce a forced linear Lagrangian system associated with our nonholonomic system. The linear system will have matrix (4.30). Then we compare the Lyapunov-Malkin approach and the energy-momentum approach for systems satisfying hypothesis $\mathrm{H} 2$.

Thus, we consider the system with matrix (4.30)

$$
\begin{aligned}
& \dot{x}=y, \\
& \dot{y}=\mathcal{A} y+(\mathcal{B}+\mathcal{C D}) x .
\end{aligned}
$$

According to Theorem 4.10, the equilibrium $x=0, y=0, z=0$ of (4.29) is stable with respect to $(x, y, z)$ and asymptotically stable with respect to $(x, y)$ if and only if the equilibrium $x=0, y=0$ of (4.31) is asymptotically stable. System (4.31) may be viewed as a linear unconstrained Lagrangian system with additional forces imposed on it. Put

$$
\begin{aligned}
C & =-\frac{1}{2}\left((\mathcal{B}+\mathcal{C D})+(\mathcal{B}+\mathcal{C D})^{t}\right), \\
F & =\frac{1}{2}\left((\mathcal{B}+\mathcal{C D})-(\mathcal{B}+\mathcal{C D})^{t}\right), \\
D & =-\frac{1}{2}\left(\mathcal{A}+\mathcal{A}^{t}\right), \\
G & =\frac{1}{2}\left(\mathcal{A}-\mathcal{A}^{t}\right) .
\end{aligned}
$$

The equations become

$$
\ddot{x}^{\alpha}=-C_{\beta}^{\alpha} x^{\beta}+F_{\beta}^{\alpha} x^{\beta}-D_{\beta}^{\alpha} \dot{x}^{\beta}+G_{\beta}^{\alpha} \dot{x}^{\beta} .
$$

These equations are the Euler-Lagrange equations with dissipation and forcing for the Lagrangian

$$
L=\frac{1}{2} \sum_{\alpha=1}^{\sigma}\left(\dot{x}^{\alpha}-G_{\beta}^{\alpha} x^{\beta}\right)^{2}-\frac{1}{2} C_{\alpha \beta} x^{\alpha} x^{\beta},
$$

with the Rayleigh dissipation function

$$
\frac{1}{2} D_{\alpha \beta} \dot{x}^{\alpha} \dot{x}^{\beta}
$$

and the nonconservative forces

$$
F_{\beta}^{\alpha} x^{\beta}
$$


Note that $D_{\alpha \beta}=\left(\mathcal{D}_{\alpha \beta b}+\mathcal{D}_{\beta \alpha b}\right) I^{a b}\left(r_{0}\right) p_{a}^{0}$.

The next theorem explains how to compute the matrices $C$ and $F$ using the amended potential of our nonholonomic system.

Theorem 4.11 The entries of the matrices $C$ and $F$ in the dissipative forced system (4.32), which is equivalent to linear system (4.31), are

$$
C_{\alpha \beta}=\frac{1}{2}\left(\nabla_{\alpha} \nabla_{\beta}+\nabla_{\beta} \nabla_{\alpha}\right) U\left(r_{0}, p_{0}\right), \quad F_{\alpha \beta}=\frac{1}{2}\left(\nabla_{\alpha} \nabla_{\beta}-\nabla_{\beta} \nabla_{\alpha}\right) U\left(r_{0}, p_{0}\right) .
$$

Proof Recall that the operators of covariant differentiation due to the transport equation are (see (3.4))

$$
\nabla_{\alpha}=\frac{\partial}{\partial r^{\alpha}}+\mathcal{D}_{a \alpha}^{c} p_{c} \frac{\partial}{\partial p_{a}}
$$

Consequently,

$$
\begin{aligned}
\nabla_{\beta} \nabla_{\alpha}= & \nabla_{\beta}\left(\frac{\partial}{\partial r^{\alpha}}+\mathcal{D}_{a \alpha}^{c} p_{c} \frac{\partial}{\partial p_{a}}\right) \\
= & \left(\frac{\partial}{\partial r^{\beta}}+\mathcal{D}_{b \beta}^{d} p_{d} \frac{\partial}{\partial p_{b}}\right)\left(\frac{\partial}{\partial r^{\alpha}}+\mathcal{D}_{a \alpha}^{c} p_{c} \frac{\partial}{\partial p_{a}}\right) \\
= & \frac{\partial^{2}}{\partial r^{\beta} \partial r^{\alpha}}+\frac{\partial}{\partial r^{\beta}}\left(\mathcal{D}_{a \alpha}^{c} \frac{\partial}{\partial p_{a}}\right) p_{c}+\mathcal{D}_{b \beta}^{d} p_{d} \frac{\partial^{2}}{\partial p_{b} \partial r^{\alpha}} \\
& \quad+\mathcal{D}_{b \beta}^{d} p_{d} \mathcal{D}_{a \alpha}^{c} \frac{\partial}{\partial p_{b}}\left(p_{c} \frac{\partial}{\partial p_{a}}\right) .
\end{aligned}
$$

Therefore, for the amended potential $U=V+\frac{1}{2} I^{a b} p_{a} p_{b}$ we obtain

$$
\begin{aligned}
\nabla_{\beta} \nabla_{\alpha} U= & \frac{\partial^{2} V}{\partial r^{\beta} \partial r^{\alpha}}+\frac{1}{2} \frac{\partial^{2} I^{a b}}{\partial r^{\beta} \partial r^{\alpha}} p_{a} p_{b}+\frac{\partial}{\partial r^{\beta}}\left(\mathcal{D}_{a \alpha}^{c} I^{a b}\right) p_{c} p_{d} \\
& +\mathcal{D}_{b \beta}^{d} \frac{\partial I^{a b}}{\partial r^{\alpha}} p_{a} p_{d}+\mathcal{D}_{b \beta}^{d} \mathcal{D}_{a \alpha}^{c} I^{a b} p_{c} p_{d}+\mathcal{D}_{b \beta}^{d} \mathcal{D}_{a \alpha}^{b} I^{a c} p_{c} p_{d}
\end{aligned}
$$

Formulae (4.22), (4.23), and (4.24) imply that

$$
\begin{aligned}
(\mathcal{B C}+\mathcal{D})_{\alpha \beta}= & \frac{\partial^{2} V}{\partial r^{\beta} \partial r^{\alpha}}\left(r_{0}\right)+\frac{1}{2} \frac{\partial^{2} I^{a b}}{\partial r^{\beta} \partial r^{\alpha}}\left(r_{0}\right) p_{a}^{0} p_{b}^{0} \\
& +\frac{\partial}{\partial r^{\beta}}\left(\mathcal{D}_{a \alpha}^{c} I^{a b}\right)\left(r_{0}\right) p_{c}^{0} p_{d}^{0}+\mathcal{D}_{b \beta}^{d} \frac{\partial I^{a b}}{\partial r^{\alpha}}\left(r_{0}\right) p_{a}^{0} p_{d}^{0} \\
& +\mathcal{D}_{b \beta}^{d} \mathcal{D}_{a \alpha}^{c} I^{a b}\left(r_{0}\right) p_{c}^{0} p_{d}^{0}+\mathcal{D}_{b \beta}^{d} \mathcal{D}_{a \alpha}^{b} I^{a c}\left(r_{0}\right) p_{c}^{0} p_{d}^{0} \\
= & \nabla_{\beta} \nabla_{\alpha} U\left(r_{0}, p_{0}\right) .
\end{aligned}
$$


Therefore

$C_{\alpha \beta}=\frac{1}{2}\left(\nabla_{\alpha} \nabla_{\beta}+\nabla_{\beta} \nabla_{\alpha}\right) U\left(r_{0}, p_{0}\right), \quad F_{\alpha \beta}=\frac{1}{2}\left(\nabla_{\alpha} \nabla_{\beta}-\nabla_{\beta} \nabla_{\alpha}\right) U\left(r_{0}, p_{0}\right)$.

Observe that the equilibrium $x=0, y=0, z=0$ of (4.29) is stable with respect to $(x, y, z)$ and asymptotically stable with respect to $(x, y)$ if and only if the equilibrium $x=0, y=0$ of the above linear Lagrangian system is asymptotically stable. The condition for stability of the equilibrium $r=r_{0}$, $p=p_{0}$ of our nonholonomic system becomes: all eigenvalues of the matrix

$$
\left(\begin{array}{cc}
0 & I \\
\nabla_{\beta} \nabla_{\alpha} U\left(r_{0}, p_{0}\right) & -\left(\mathcal{D}_{\beta \alpha b} I^{a b}\left(r_{0}\right)+\mathcal{B}_{\alpha \beta}^{a}\left(r_{0}\right)\right) p_{a}^{0}
\end{array}\right)
$$

have negative real parts.

If the transport equation is integrable (hypothesis H2), then the operators $\nabla_{\alpha}$ and $\nabla_{\beta}$ commute, and the corresponding linear Lagrangian system (4.32) has no nonconservative forces imposed on it. In this case the sufficient conditions for stability are given by the Thompson theorem (Thompson and Tait [1987], Chetaev[1959]): the equilibrium $x=0$ of (4.32) is asymptotically stable if the matrices $C$ and $D$ are positive definite. These conditions are identical to the energy-momentum conditions for stability obtained in Theorem 4.7. Notice that if $C$ and $D$ are positive definite, then matrix (4.30) is positive definite. This implies that the matrix $A$ in Theorem 4.4 has spectrum in the left half plane. Further, our coordinate transformations here give the required form for the nonlinear terms of Theorem 4.4. Therefore, the above analysis shows that hypothesis H3 implies the hypotheses of Theorem 4.4.

Remark. On the other hand (cf. Chetaev [1959]), if the matrix $C$ is not positive definite (and thus the equilibrium of the system $\ddot{x}=-C x$ is unstable), and the matrix $D$ is degenerate, then in certain cases the equilibrium of the equations $\ddot{x}=-C x-D \dot{x}+G \dot{x}$ may be stable. Therefore, the conditions of Theorem 4.7 are sufficient, but not necessary.

\subsection{The Rattleback}

Here we outline the stability theory of the rattleback to illustrate the results discussed above. The details may be found in Karapetyan [1980, 1981] and Markeev [1992]. 
Recall that the Lagrangian and the constraints are

$$
\begin{aligned}
L= & \frac{1}{2}\left[A \cos ^{2} \psi+B \sin ^{2} \psi+m\left(\gamma_{1} \cos \theta-\zeta \sin \theta\right)^{2}\right] \dot{\theta}^{2} \\
& +\frac{1}{2}\left[\left(A \sin ^{2} \psi+B \cos ^{2} \psi\right) \sin ^{2} \theta+C \cos ^{2} \theta\right] \dot{\phi}^{2} \\
& +\frac{1}{2}\left(C+m \gamma_{2}^{2} \sin ^{2} \theta\right) \dot{\psi}^{2}+\frac{1}{2} m\left(\dot{x}^{2}+\dot{y}^{2}\right) \\
& +m\left(\gamma_{1} \cos \theta-\zeta \sin \theta\right) \gamma_{2} \sin \theta \dot{\theta} \dot{\psi}+(A-B) \sin \theta \sin \psi \cos \psi \dot{\theta} \dot{\phi} \\
& +C \cos \theta \dot{\phi} \dot{\psi}+m g\left(\gamma_{1} \sin \theta+\zeta \cos \theta\right)
\end{aligned}
$$

and

$$
\dot{x}=\alpha_{1} \dot{\theta}+\alpha_{2} \dot{\psi}+\alpha_{3} \dot{\phi}, \quad \dot{y}=\beta_{1} \dot{\theta}+\beta_{2} \dot{\psi}+\beta_{3} \dot{\phi},
$$

where the terms were defined in $\S 1.6$.

Using the Lie algebra element corresponding to the generator $\xi_{Q}=$ $\alpha_{3} \partial_{x}+\beta_{3} \partial_{y}+\partial_{\phi}$ we find the nonholonomic momentum to be

$$
\begin{aligned}
p=I(\theta, \psi) \dot{\phi}+\left[(A-B) \sin \theta \sin \psi \cos \psi-m\left(\gamma_{1} \sin \theta+\zeta \cos \theta\right) \gamma_{2}\right] \dot{\theta} \\
+\left[C \cos \theta+m\left(\gamma_{2}^{2} \cos \theta+\gamma_{1}\left(\gamma_{1} \cos \theta-\zeta \sin \theta\right)\right)\right] \dot{\psi}
\end{aligned}
$$

where

$$
\begin{aligned}
I(\theta, \psi)=\left(A \sin ^{2} \psi+B \cos ^{2} \psi\right) & \sin ^{2} \theta+C \cos ^{2} \theta \\
& +m\left(\gamma_{2}^{2}+\left(\gamma_{1} \cos \theta-\zeta \sin \theta\right)^{2}\right) .
\end{aligned}
$$

The amended potential becomes

$$
U=\frac{p^{2}}{2 I(\theta, \psi)}-m g\left(\gamma_{1} \sin \theta+\zeta \cos \theta\right)
$$

The relative equilibria of the rattleback are

$$
\theta=\theta_{0}, \quad \psi=\psi_{0}, \quad p=p_{0}
$$

where $\theta_{0}, \psi_{0}$, and $p_{0}$ satisfy the conditions

$$
\begin{aligned}
& m g\left(\gamma_{1} \cos \theta_{0}-\zeta \sin \theta_{0}\right) I^{2}\left(\theta_{0}, \psi_{0}\right) \\
& +\left[\left(A \sin ^{2} \psi_{0}+B \cos ^{2} \psi_{0}-C\right) \sin \theta_{0} \cos \theta_{0}\right. \\
& \left.-m\left(\gamma_{1} \cos \theta_{0}-\zeta \sin \theta_{0}\right)\left(\gamma_{1} \sin \theta_{0}+\zeta \cos \theta_{0}\right)\right] p_{0}^{2}=0, \\
& m g \gamma_{2} I^{2}\left(\theta_{0}, \psi_{0}\right)+\left[(A-B) \sin \theta_{0} \sin \psi_{0} \cos \psi_{0}\right. \\
& \left.-m \gamma_{2}\left(\gamma_{1} \sin \theta_{0}+\zeta \cos \theta_{0}\right)\right] p_{0}^{2}=0,
\end{aligned}
$$


which are derived from $\nabla_{\theta} U=0, \nabla_{\psi} U=0$.

In particular, consider the relative equilibria

$$
\theta=\frac{\pi}{2}, \quad \psi=0, \quad p=p_{0},
$$

that represent the rotations of the rattleback about the vertical axis of inertia. For such relative equilibria $\xi=\zeta=0$, and therefore the conditions for existence of relative equilibria are trivially satisfied with an arbitrary value of $p_{0}$. Omitting the computations of the linearized equations for the rattleback, which have the form discussed in $§ 4.6$ (see Karapetyan [1980] for details), and the corresponding characteristic polynomial, we just state here the Routh-Hurwitz conditions for all eigenvalues to have negative real parts:

$$
\begin{array}{r}
\left(R-P \frac{p_{0}^{2}}{B^{2}}\right) \frac{p_{0}^{2}}{B^{2}}>S>0, \\
(A-C)\left(r_{2}-r_{1}\right) p_{0} \sin \alpha \cos \alpha>0 .
\end{array}
$$

If these conditions are satisfied, then the relative equilibrium is stable, and it is asymptotically stable with respect to $(\theta, \dot{\theta}, \psi, \dot{\psi})$.

In the above formulae $r_{1}$ and $r_{2}$ stand for the radii of curvature of the body at the contact point, $\alpha$ is the angle between horizontal inertia axis $\xi$ and the $r_{1}$-curvature direction, and

$$
\begin{aligned}
P= & \left(A+m a^{2}\right)\left(C+m a^{2}\right), \\
R= & {\left[\left(A+C-B+2 m a^{2}\right)^{2}\right.} \\
& \left.-\left(A+C-B+2 m a^{2}\right) m a\left(r_{1}+r_{2}\right)+m^{2} a^{2} r_{1} r_{2}\right] \frac{p_{0}^{2}}{B^{2}} \\
& -\left[(A-B) \frac{p_{0}^{2}}{B^{2}}+m\left(a-r_{1} \sin ^{2} \alpha-r_{2} \cos ^{2} \alpha\right)\left(g+a \frac{p_{0}^{2}}{B^{2}}\right)\right]\left(A+m a^{2}\right) \\
& -\left[(C-B) \frac{p_{0}^{2}}{B^{2}}+m\left(a-r_{2} \sin ^{2} \alpha-r_{1} \cos ^{2} \alpha\right)\left(g+a \frac{p_{0}^{2}}{B^{2}}\right)\right]\left(C+m a^{2}\right), \\
S= & (A-B)(C-B) \frac{p_{0}^{4}}{B^{4}}+m^{2}\left(a-r_{1}\right)\left(a-r_{2}\right)\left(g+m \frac{p_{0}^{2}}{B^{2}}\right)^{2} \\
& +m \frac{p_{0}^{2}}{B^{2}}\left(g+a \frac{p^{2}}{B^{2}}\right)\left[A\left(a-r_{1} \cos ^{2} \alpha-r_{2} \sin ^{2} \alpha\right)\right. \\
& \left.+C\left(a-r_{1} \sin ^{2} \alpha-r^{2} \cos ^{2} \alpha\right)-B\left(2 a-r_{1}-r_{2}\right)\right] .
\end{aligned}
$$

Condition (4.33) imposes restrictions on the mass distribution, the magnitude of the angular velocity, and the shape of the rattleback only. Condition (4.34) distinguishes the direction of rotation corresponding to the stable 
relative equilibrium. The rotation will be stable if the largest (smallest) principal inertia axis precedes the largest (smallest) direction of curvature at the point of contact.

The rattleback is also capable of performing stationary rotations with its center of mass moving at a constant rate along a circle. A similar argument gives the stability conditions in this case. The details may be found in Karapetyan [1981] and Markeev [1992].

\subsection{Conclusions}

We have given a general energy-momentum method for analyzing the stability of relative equilibria of a large class of nonholonomic systems. We have also shown that for systems to which the classical Lyapunov-Malkin theorem applies, one can interpret and even verify the hypotheses in terms of definiteness conditions on the second variation of energy-momentum functions. We have also studied stability of some systems (in the pure transport case) for which energetic arguments give stability, but the hypotheses of the Lyapunov-Malkin theorem fail (because eigenvalues are on the imaginary axis).

As indicated in the text, not all nonholonomic systems satisfy the assumptions made in this paper (and of the Lyapunov-Malkin theorem) and we intend to consider these in a forthcoming publication.

\section{Acknowledgments}

We would like to thank J. Burdick, P. Crouch, W. Koon, P. Krishnaprasad, and R. Murray for helpful comments and suggestions.

\section{References}

Abraham, R. \& J.E. Marsden [1978] Foundations of Mechanics. Second Edition, Addison-Wesley.

Appel, P. [1900] Sur l'intégration des équations du mouvement d'un corps pesant de révolution roulant par une arête circulaire sur un plan horizontal; cas parficulier du cerceau. Rendiconti del circolo matematico di Palermo 14, 1-6.

Arnold, V.I. [1966] Sur la geometrie differentielle des groupes de Lie de dimension infinie et ses applications a a l'hydrodynamique des fluids parfaits. Ann. Inst. Fourier, Grenoble 16, 319-361. 
Arnold, V.I. [1988] Dynamical Systems III. Springer-Verlag, New York.

Arnold, V.I. [1989] Mathematical Methods of Classical Mechanics. Second Edition, Graduate Texts in Mathematics 60, Springer-Verlag.

Bates, L. \& J. Sniatycki [1993] Nonholonomic Reduction. Reports on Math. Phys. 32, 99-115.

Bloch, A.M. \& P. Crouch [1992] On the Dynamics and Control of Nonholonomic Systems on Riemannian Manifolds. Proceedings of NOLCOS '92, Bordeaux, 368-372.

Bloch, A.M., P.S. Krishnaprasad, J.E. Marsden \& G. Sánchez de Alvarez [1992] Stabilization of Rigid Body Dynamics by Internal and External Torques. Automatica 28, 745-756.

Bloch, A.M., M. Reyhanoglu \& H. McClamroch [1992] Control and Stabilization of Nonholonomic Systems. IEEE Trans. Aut. Control 37, 1746-1757.

Bloch, A.M., P.S. Krishnaprasad, J.E. Marsden, \& T.S. Ratiu [1994] Dissipation Induced Instabilities. Ann. Inst. H. Poincaré, Analyse Nonlineare 11, 37-90.

Bloch, A.M. \& P. Crouch [1995] Nonholonomic Control Systems on Riemannian Manifolds. SIAM J. on Control 37, 126-148.

Bloch, A.M., P.S. Krishnaprasad, J.E. Marsden, \& R. Murray [1996] Nonholonomic Mechanical Systems with Symmetry. Arch. Rat. Mech. An. 136, 21-99.

Bloch, A.M., P.S. Krishnaprasad, J.E. Marsden, \& T.S. Ratiu [1996] The Euler-Poincaré Equations and Double Bracket Dissipation. Comm. Math. Phys. 175, 1-42.

Bondi, H. [1986] The Rigid Body Dynamics of Unidirectional Spin. Proc. Roy. Soc. Lon. 405, 265-274.

Brockett, R.W. \& L. Dai [1992] Nonholonomic Kinematics and the Role of Elliptic Functions in Constructive Controllability. In Nonholonomic Motion Planning, eds. Z. Li \& J. F. Canny, Kluwer, 1-22, 1993.

Burdick, J., B. Goodwine, \& J. Ostrowski [1994] The Rattleback Revisited. California Institute of Technology, preprint. 
Carr J. [1981] Applications of Centre Manifold Theory. Springer-Verlag, New York.

Chaplygin, S.A. [1897a] On the Motion of a Heavy Body of Revolution on a Horizontal Plane (in Russian). Physics Section of the Imperial Society of Friends of Physics, Anthropology and Ethnographics, Moscow 9, 10-16. (Reproduced in Chaplygin [1954], 413-425).

Chaplygin, S.A. [1897b] On Some Feasible Generalization of the Theorem of Area, with an Application to the Problem of Rolling Spheres (in Russian). Mat. Sbornik XX, 1-32. (Reproduced in Chaplygin [1954], 434-454).

Chaplygin, S.A. [1903] On a Rolling Sphere on a Horizontal Plane (in Russian). Mat. Sbornik XXIV, 139-168. (Reproduced in Chaplygin [1949], 72-99 and Chaplygin [1954], 455-471).

Chaplygin, S.A. [1911] On the Theory of the Motion of Nonholonomic Systems. Theorem on the Reducing Factor (in Russian). Mat. Sbornik XXVIII, 303-314. (Reproduced in Chaplygin [1949], 28-38 and Chaplygin [1954], 426-433).

Chaplygin, S.A. [1949] Analysis of the Dynamics of Nonholonomic Systems (in Russian), Classical Natural Sciences, Moscow.

Chaplygin, S.A. [1954] Selected Works on Mechanics and Mathematics. (in Russian), State Publ. House, Technical-Theoretical Literature, Moscow.

Chern, S.J. \& J.E. Marsden [1990] A Note on Symmetry and Stability for Fluid Flows, Geo. Astro. Fluid. Dyn. 51, 1-4.

Chetaev, N.G. [1959] The Stability of Motion. Pergamon Press, New York.

Chow, S.N. \& J.K. Hale [1982] Methods of Bifurcation Theory. Springer, New York.

Cushman, R.; Hermans, J.; Kemppainen, D. [1996] The Rolling Disc. In Nonlinear Dynamical Systems and Chaos (Groningen, 1995), 21-60, Progr. Nonlinear Differential Equations Appl. 19, Birkhauser, Basel, 1996.

Getz, N.H. \& J. E. Marsden [1994] Symmetry and Dynamics of the Rolling Disk. Preprint 630, Center for Pure and Applied Mathematics, UC Berkeley. 
Getz, N.H. \& J. E. Marsden [1995] Control for an Autonomous Bicycle. International Conference on Robotics and Automation, IEEE, Nagoya, Japan, May, 1995.

Hermans, J. [1995a], Rolling Rigid Bodies, with and without Symmetries. $\mathrm{PhD}$ Thesis, University of Utrecht.

Hermans, J. [1995b] A Symmetric Sphere Rolling on a Surface. Nonlinearity 8, 493-515.

Hamel, G. [1904] Die Lagrange-Eulerschen Gleichungen der Mechanik. Z. für Mathematik u. Physik 50, 1-57.

Jurdjevic, V. [1993] The Geometry of the Plate-Ball Problem. Arch. Rat. Mech. An. 124, 305-328.

Karapetyan, A.V. [1980] On the Problem of Steady Motions of Nonholonomic Systems. Appl. Math. Mech. 44, 418-426.

Karapetyan, A.V. [1981] On Stability of Steady State Motions of a Heavy Solid Body on an Absolutely Rough Horizontal Plane. Appl. Math. Mech. 45, 604-608.

Karapetyan, A.V. [1983] Stability of Steady Motions of Systems of a Certain Type. Mechanics of Solids 18, 41-47.

Kelly, S.D. \& R.M. Murray [1995] Geometric Phases and Robotic Locomotion. Journal of Robotic Systems (to appear). Also available as Caltech technical report CIT/CDS 94-014.

Koiller, J. [1992] Reduction of Some Classical Nonholonomic Systems with Symmetry. Arch. Rat. Mech. An. 118, 113-148.

Koon, W.S. \& J.E. Marsden [1997a] Optimal Control for Holonomic and Nonholonomic Mechanical Systems with Symmetry and Lagrangian Reduction. SIAM J. Control and Optim. 35, 901-929.

Koon, W.S. \& J.E. Marsden [1997b] The Hamiltonian and Lagrangian Approaches to the Dynamics of Nonholonomic Systems. Reports on Math Phys. 40, 21-62.

Koon, W.S. \& J.E. Marsden [1997c] The Poisson Reduction of Nonholonomic Mechanical Systems. Reports on Math Phys (to appear). 
Korteweg, D. [1899] Ueber eine ziemlich verbreitete unrichtige Behandlungsweise eines Problemes der rollenden Bewegung und insbesondere über kleine rollende Schwingungen um eine Gleichgewichtslage. Nieuw Archiefvoor Wiskunde. 4, 130-155.

Kozlov, V.V. \& N.N. Kolesnikov [1978] On Theorems of Dynamics. Appl. Math. Mech. 42, 28-33.

Krishnaprasad, P.S. [1989] Eulerian Many-Body Problems. Cont. Math. AMS 97, 187-208.

Lewis, A., J.P. Ostrowski, R.M. Murray \& J. Burdick [1994] Nonholonomic Mechanics and Locomotion: the Snakeboard Example. IEEE Intern. Conf. on Robotics and Automation.

Lyapunov, A.M. [1992] The General Problem of the Stability of Motion. translated and edited by A.T. Fuller. London, Washington, DC : Tayor \& Francis.

Malkin, I.G. [1938] On the Stability of Motion in a Sense of Lyapunov (in Russian). Mat. Sbornik XXXV, 47-101.

Markeev A.P. [1983] On Dynamics of a Solid on an Absolutely Rough Plane. Appl. Math. Mech. 47, 473-478.

Markeev A.P. [1992] Dynamics of a Body Being Contiguous to a Rigid Surface. (In Russian), Moscow, Nauka.

Marsden, J.E., P.S. Krishnaprasad \& J.C. Simo (eds.) [1989] Dynamics and Control of Multibody Systems Contemporary Mathematics, Am. Math. Soc. 97.

Marsden, J.E., R. Montgomery \& T.S. Ratiu [1990] Reduction, Symmetry, and Phases in Mechanics. Memoirs AMS 436.

Marsden, J.E. [1992], Lectures on Mechanics. London Mathematical Society Lecture Note Series 174, Cambridge University Press.

Marsden, J.E. \& J. Scheurle [1993a] Lagrangian Reduction and the Double Spherical Pendulum. ZAMP 44, 17-43.

Marsden, J.E. \& J. Scheurle [1993b] The Reduced Euler-Lagrange Equations. Fields Institute Comm. 1, 139-164. 
Marsden, J.E. \& T.S. Ratiu [1994] An Introduction to Mechanics and Symmetry. Texts in Appl. Math. 17, Springer-Verlag.

Milnor, J. [1963] Morse Theory, Princeton University Press, Princeton, NJ

Neimark, Ju. I. \& N.A. Fufaev [1966] On Stability of Stationary Motions of Holonomic and Nonholonomic Systems. Appl. Math. Mech. 30, 293-300.

Neimark, Ju. I. \& N.A. Fufaev [1972] Dynamics of Nonholonomic Systems. Translations of Mathematical Monographs, AMS 33.

O'Reilly, O.M. [1996] The Dynamics of Rolling Disks and Sliding Disks. Nonlinear Dynamics 10, 287-305.

Ostrowski, J. [1995] Geometric Perspectives on the Mechanics and Control of Undulatory Locomotion. $\mathrm{PhD}$ dissertation, California Institute of Technology.

Ostrowski, J., J. W. Burdick, A. D. Lewis \& R. M. Murray [1995] The Mechanics of Undulatory Locomotion: The Mixed Kinematic and Dynamic Case. IEEE Intern. Conf. on Robotics and Automation, 19451951.

Pascal, M. [1983] Asymptptic Solution of the Equations of Motion for a Celtic Stone. Appl. Math. Mech. 47, 269-276.

Pascal, M. [1986] The Use of the Method of Averaging to Study Nonlinear Oscillations of the Celtic Stone. Appl. Math. Mech. 50, 520-522.

Poincaré, H. [1901] Sur une forme nouvelle des equations de la mecanique. CR Acad. Sci. 132, 369-371.

Rosenberg, R.M. [1977] Analytical Dynamics of Discrete Systems. Plenum Press, NY.

Routh, E.J. [1860] Treatise on the Dynamics of a System of Rigid Bodies. MacMillan, London.

Simo, J.C., D.R. Lewis \& J.E. Marsden [1991] Stability of Relative Equilibria I: The Reduced Energy-Momentum Method. Arch. Rat. Mech. An. 115, 15-59.

Smale, S. [1970] Topology and Mechanics. Inv. Math. 10, 305-331, 11, 45-64. 
Sumbatov, A.S. [1992] Developments of Some of Lagrange's Ideas in the Works of Russian and Soviet Mechanicians. La Mécanique Analytique de Lagrange et Son héritage, Atti della Accademia delle Scienze di Torino, Suppl 2, vol 126, 169-200.

Thompson, W. \& P.G. Tait [1987], Treatise on Natural Philosphy. Cambridge University Press.

Tsakiris, D.P. [1995] Motion Control and Planning for Nonholonomic Kinematic Chains. PhD Thesis, Systems Research Institute, University of Maryland.

Vershik, A.M. \& L.D. Faddeev [1981] Lagrangian Mechanics in Invariant Form. Sel. Math. Sov. 1, 339-350.

Vershik, A.M. \& V.Ya. Gershkovich [1994] Nonholonomic Dynamical Systems, Geometry of Distributions and Variational Problems. Dynamical Systems VII, V.I. Arnold and S.P. Novikov, eds., 1-81. SpringerVerlag, New York.

Vierkandt, A. [1892] Über gleitende und rollende Bewegung. Monatshefte der Math. und Phys. III, 31-54.

Walker, G.T. [1896] On a Dynamical Top. Quart. J. Pure Appl. Math. 28, 175-184.

Wang, L.S. \& P.S. Krishnaprasad [1992] Gyroscopic Control and Stabilization. J. Nonlinear Sci. 2, 367-415.

Yang, R. [1992] Nonholonomic Geometry, Mechanics and Control. PhD Thesis, Systems Research Institute, University of Maryland.

Yang, R., P.S. Krishnaprasad, \& W. Dayawansa [1993] Chaplygin Dynamics and Lagrangian Reduction. Proc. 2nd Int. Cong. on Nonlinear Mechanics, W-Z. Chien, Z.H. Guo \& Y.Z. Guo, eds., Peking University Press, 745-749.

Zenkov, D.V. [1995] The Geometry of the Routh Problem. J. Nonlinear Sci. 5, 503-519. 